\title{
FITOGEOGRAFÍA Y VEGETACIÓN DEL SECTOR ALJÍBICO (CÁDIZ-MÁLAGA, ESPAÑA)
}

\author{
Andrés V. PÉREZ LATORRE, Antonio GALÁN DE MERA, \\ Ulrich DEIL y Baltasar CABEZUDO
}

RESUMEN. Fitogeografía y vegetación del sector Aljíbico (Cádiz-Málaga, España). Se analiza la flora y vegetación del sur de la Península Ibérica (Andalucía, provincias de Cádiz y Málaga) con el fin de delimitar y subsectorizar la unidad fitogeográfica Aljíbica.

Hemos reconocido 4 subsectores en base a la bioclimatología, flora y vegetación: Aljíbico, Algecireño, Marbellí y Sidonense. Incluímos el sector Aljíbico en la provincia Tingitano-Onubo-Algarviense. Dicha provincia forma parte de la superprovincia Iberomarroquí-Atlántica que enlaza las regiones Eurosiberiana y Macaronésica con la Mediterránea.

Las series de vegetación reconocidas en el sector Aljíbico han sido las siguientes: alcornocales de Myrto-Querceto suberis S y Teucrio-Querceto suberis S, quejigares de Rusco-Querceto canariensis S, robledales de Cytiso-Querceto pyrenaicae $\mathrm{S}$, acebuchales de Tamo-Oleeto sylvestris $\mathrm{S}$, algarrobales de Clematido-Ceratonieto siliquae S, alisedas de Arisaro-Alneto glutinosae S, hojaranzales de FranguloRhododendreto baetici S, fresnedas de Ficario-Fraxineto angustifoliae S, brezales higrófilos de GenistoEriceto ciliaris S, choperas de Crataego-Populeto albae S, tarajales de Polygono-Tamariceto africanae S y de Agrostio-Tamariceto canariensis S y adelfares de Rubo-Nerieto oleandri S.

Se lectotipifican Tamo-Oleetum sylvestris oleetosum y fraxinetosum angustifoliae y PolygonoTamaricetum africanae aretosum italici. Como nuevos sintaxones se proponen Crataego-Populetum albae, Rusco-Quercetum canariensis quercetosum broteroi, Asparago-Calicotometum villosae juniperetosum turbinatae, Equiseto-Salicetum pedicellatate salicetosum albae y Asperulo-Ulicetum scabri anthyllidetosum cytisoidis. Se describen nueve comunidades caracterizadas cada una por: Juniperus oxycedrus, Quercus lusitanica e Ilex aquifolium, Dianthus lusitanus, Potamogeton nodosus, Potamogeton pectinatus, Myriophyllum alterniflorum, Callitriche stagnalis, Callitriche regis-jubae y Ranunculus tripartitus. Se cita como nueva serie para el subcontinente europeo Cytiso-Querceto pyrenaicae $\mathrm{S}$ y se propone una nueva serie, Crataego-Populeto albae S. Se incluye la alianza Campanulion velutinae en Tinguarrenalia siculae a la cual se le asigna el rango de suborden.

Palabras clave. Fitogeografía, Cádiz, Málaga, España, sector Aljíbico, vegetación.

ABSTRACT. Phytogeography and vegetation of the Aljibico sector (Cadiz-Malaga, Spain). In this study, the flora and vegetation of the southern Iberian Peninsula (Cadiz and Malaga provinces, Andalusia, Spain) are analized for delimitating and dividing the phytogeographical unit of the Aljibe mountains. This unit includes most of the Gaditanian Peninsula. It is characterized in its physical conditions by the geomorphologic «flysch» of the Campo de Gibraltar and the siliceous base of the Serranía de Ronda, and in its climatic aspect by an atlantic temperature regime and by a mediterranean precipitation regime.

As a result, the Aljibico sector is delimited as follows: towards the West by the dune systems of the Cadiz Gulf (Onubense sector), towards the South by he Gibraltar Strait and the Tingitanian Peninsula, towards the East by the Alboran Sea and the Guadalhorce Valley (Malacitano-Axarquiense sector) and towards the North by the Serranía deRonda (Bermejense and Rondeño sectors towards NE) and the Guadalquivir Valley (Hispalense sector towards NW). 
Based on the chorology of the flora and the vegetation and upon the abiotic conditions, four subsectors are considered: Aljibico, the central part with siliceous sandstones, humid-hyperhumid ombroclimate and some continental characteristics; Algecireño, the southern area with similar geology and ombroclimate but with an oceanic precipitation regime and frequent orographic fogs; Marbellí, the eastern area with saltes and micaschists and a subhumid-humid ombroclimate; and Sidonense, the western area with a heterogeneous geology of sandstones, limestones, vertic argils, sands, a dry-subhumid ombroclimate and a thermic continental regime. The Aljibico sector is included in the Tingitano-Onubo-Algarviense province. This province reaches from Aveiro (Portugal) to Bajo Loukkos (Morocco). It is also part of the IberomarroquiAtlantica superprovince that connects the Eurosiberian and the Macaronesian areas through the Mediterranean region.

The vegetation series considered in the Aljibico sector are the following: Myrto-Querceto suberis $\mathrm{S}$, Teucrio-Querceto suberis S, Rusco-Querceto canariensis S, Cytiso-Querceto pyrenaicae S, Tamo-Oleeto sylvestris S, Clematido-Ceratonieto siliquae S, Arisaro-Alneto glutinosae S, Frangulo-Rhododendreto baetici S, Ficario-Fraxineto angustifoliae S, Genisto-Ericeto ciliaris S, Crataego-Populeto albae S, Polygono-Tamariceto africanae S, Agrostio-Tamariceto canariensis S and Rubo-Nerieto oleandri S. Likewise has plenty of rupiculous, dunes and aquatic plant communities, while the swamp communities are very limited.

Tamo-Oleetum sylvestris (oleetosum and fraxinetosum angustifoliae) and Polygono-Tamaricetum africanae aretosum italici are lectotypified. As new syntaxa, Crataego-Populetumalbae, Rusco-Quercetum canariensis quercetosum broteroi, Asparago-Calicotometum villosae juniperetosum turbinatae, EquisetoSalicetum pedicellatate salicetosum albae and Asperulo-Ulicetum scabri anthyllidetosum cytisoidis are proposed. Furthermore, nine dominance communities are described here. They are characterized by the following species: Juniperus oxycedrus, Quercus lusitanica-Ilex aquifolium, Dianthus lusitanus, Potamogeton nodosus, Potamogeton pectinatus, Myriophyllum alterniflorum, Callitriche stagnalis, Callitriche regis-jubae and Ranunculus tripartitus. Cytiso-Querceto pyrenaicae $\mathrm{S}$ is a new record for Europe. Crataego-Populeto albae $\mathrm{S}$ is proposed as a new series of vegetation. The suborder status for Tinguarrenalia siculae is proposed. The alliance Campanulion velutinae is included in this suborder.

Key words. Phytogeography, Cadiz, Malaga, Spain, Aljibico sector, vegetation.

\section{INTRODUCCIÓN}

El sector Aljíbico pertenece administrativamente a las provincias de Cádiz y Málaga (Andalucía, sur de España) y es el territorio más meridional de la Península Ibérica. Esta posición le confiere una climatología peculiar, de carácter oceánico, y además una litología, flora, vegetación e historia paleofitogeográfica (Valdés, 1996) muy semejantes a la Península Tingitana en el norte de Marruecos. Se trata de una de las áreas con mayor riqueza biológica en el ámbito de la Unión Europea (Gil Jiménez et al., 1996); su ubicación «puente» entre Africa y Europa conlleva una elevada biodiversidad florística y fitocenológica (Valdés et al., 1987; Pérez Latorre, 1993; Galán de Mera, 1993).
Sus límites, hasta ahora, han quedado dibujados según el areal de las sierras del Aljibe y del Campo de Gibraltar; una comarca integrada por un conjunto de elevaciones formadas por areniscas silíceas y colinas margosas que tienen primero dirección norte a sur, y se flexionan después cerca de Tarifa, formando alineaciones E-W (Ceballos y Martín Bolaños, 1930; Asensi y Díez Garretas in Peinado y Rivas-Martínez, 1987; RivasMartínez, 1988).

Los objetivos de este trabajo consisten en establecer los límites del sector Aljíbico y describir la variabilidad de sus subunidades, teniendo en cuenta la diversidad de sustratos, la riqueza de taxones y sintaxones característicos o endémicos, las series de vegetación y las relaciones existentes con el norte de Africa. 


\section{MATERIAL Y MÉTODOS}

\section{Geología}

Desde el punto de vista geológico se trata de un territorio muy heterogéneo con tres unidades básicas: la campiña de Medina Sidonia, las sierras del Aljibe y del campo de Gibraltar, y las áreas paleozoicas cuarcíticas que se extienden entre Estepona y Fuengirola penetrando por el Valle del río Genal (Málaga).

La campiña de Medina Sidonia se extiende desde el río Barbate hasta el río Guadalete y se caracteriza sobre todo por la presencia de arcillas y margas con areniscas y calizas organógenas. Las areniscas silíceas aparecen en grandes areales que sobresalen por encima del Trías margo-yesífero subbético. Al sur de la campiña afloran, de nuevo, materiales silíceos donde abundan los conglomerados. Las calizas organógenas también son especialmente significativas, y aparecen representadas como mesas calizas alineadas en dirección NE-SW. Las más grandes del territorio son donde están asentadas las poblaciones de Benalup y Medina Sidonia.

En las sierras del Aljibe y del campo de Gibraltar es donde las areniscas silíceas alcanzan una mayor extensión con grandes relieves como El Picacho (882 m), Pico del Aljibe (1091 m), Puerto de la Yegua (915) m y Tajo de la Corza (840 m) que dominan la zona septentrional y meridional del área estudiada.

El sector oriental, hasta Fuengirola y valle de Genal, está constituído sobre todo por materiales antiguos metamorfizados, representantes del zócalo prealpino y, en parte, herciniano (pizarras, filitas, cuarcitas, micaesquistos y gneises).

La situación geológica del sector Aljíbico, desde Jerez de la Frontera a Fuengirola, es muy semejante a la de la Península Tingitana (norte de Africa): con arcillas, margas y areniscas silíceas, y las primeras estribaciones del Rif con materiales metamórficos más antiguos (Didon et al., 1973; Gutiérrez et al., 1991).
En algunos puntos del interior, el relieve se encuentra interrumpido por promontorios calizos de origen jurásico. Los más significativos son Peña Arpada y la peña del Almed (ambos en Alcalá de los Gazules, Cádiz), el peñón de Gibraltar, la sierra de la Utrera (Manilva, Málaga), y la sierra Crestellina (Casares, Málaga).

\section{Bioclimatología}

Para modelizar el clima y relacionarlo con la vegetación hemos utilizado el índice de termicidad (Rivas-Martínez, 1987) y los ombrotipos (Rivas-Martínez et al., 1991). Los datos meteorológicos para su cálculo fueron obtenidos del Instituto Nacional de Meteorología (España).

\section{La flora y la vegetación}

Para la caracterización corológica de los taxones y sintaxones del territorio estudiado, hemos empleado las principales fuentes sobre flora y vegetación del sur de la Península Ibérica, norte de Africa y otras áreas atlánticas europeas (Willkomm \& Lange, 1861-1880; Ceballos y Martín Bolaños, 1930; Ceballos y Vicioso, 1933; Jahandiez \& Maire, 1931-1934; Maire, 1953-1987; Quézel, 1957, 1978; Dupont, 1962; Tutin et al., 1964-1980; Dahlgren \& Lassen, 1972; Rivas-Martínez et al., 1980; Greuter et al., 1984-1989; Gil et al., 1985; Castroviejo et al., 1986-1993; Valdés et al., 1987; Pichi Sermolli et al., 1988; RivasMartínez, 1988; Bolós et al., 1990; Oberdorfer, 1990; Géhu, 1991; Rivas-Martínez et al., 1991; Nieto et al., 1990-1991; Pérez Latorre et al., 1993-1994; Carazo Montijano y Fernández López, 1994; Deil, 1994; Nezadal et al., 1994 y Galán de Mera y Vicente Orellana, 1996).

Para el tratamiento de la vegetación seguimos el criterio unidireccional de las series de vegetación (Rivas-Martínez, 1987). La nomenclatura de los taxones está basada en Tutin et al. (1964-1980), Greuter et al. (19841989), Castroviejo et al. (1986-1993) y Valdés 
et al. (1987), excepto en los siguientes casos: Asphodelus roseus Humbert \& Maire, Brachypodium sylvaticum (Hudson) Beauv. var. gaditanum (Talavera) Galán de Mera, Carex elata All. subsp. mauritanica (Boiss. \& Reuter) Galán de Mera, Chamaepeuce abylense Pau y Font Quer, Cytisus striatus (Hill.) Rothm. subsp. welwitschii (Boiss. \& Reuter) RivasMartínez, Dechampsia stricta (Gay) Hackel, Echinospartum algibicum Talavera y Aparicio, Festuca baetica Hackel ex Aschers. \& Graebn., Genista hirsuta Vahl subsp. lanuginosa (Spach) Nyman, Hypochaeris radicata L. subsp. platylepis (Boiss.) Jahandiez \& Maire, Lavandula luisieri (Rozeira) Rivas-Martínez, Lotus glareosus Boiss. \& Reuter var. villosus Boiss. \& Reuter, Micromeria graeca (L.) Bentham ex Reichenb. subsp. micrantha (Brot.) Rivas-Martínez, Díaz y Fernández-González, Nothobartsia aspera (Brot.) Bolliger \& Molau, Quercus broteroi (Coutinho) Rivas-Martínez y Sáenz, Rhodanthemum hosmariense (Ball) Vogt, $R$. laouense Vogt, Rhododendron ponticum L., Silene rosulata Soy.-Will. \& Godron, Sonchus fragilis Cav., Stemmacantha longifolia (Hoffmanns. \& Link) Dittrich var. ericeticola (Font Quer) Dittrich, Teucrium afrum (Emb. \& Maire) Pau y Font Quer subsp. rubriflorum (Pau y Font Quer) Castroviejo y Bayen.

\section{RESULTADOS}

\section{A. Bioclimatología y bioindicadores}

De los seis pisos bioclimáticos existentes en la Región Mediterránea solamente aparecen en el territorio el termo y mesomediterráneo. Dentro de ambos es posible reconocer horizontes o subpisos en función de taxones o sintaxones bioindicadores: termomediterráneo inferior y superior y mesomediteráneo inferior y medio.

Consideramos características del termomediterráneo en sentido amplio a
Acanthus mollis subsp. platyphyllos, Asparagus albus, Aristolochia baetica, Armeria hirta, Bellis rotundifolia, Calicotome villosa, Ceratonia siliqua, Chamaerops humilis, Osyris quadripartita, Rubia agostinhoi, Silene tomentosa y Ulex baeticus subsp. scaber. Son exclusivas del subpiso inferior Arenaria emarginata, Culcita macrocarpa, Cytisus striatus subsp. welwitschii, Festuca arundinacea subsp. atlantigena, Scrophularia laevigata y Psilotum nudum.

Como especies mesomediterráneas en sentido amplio destacamos Daphne laureola subsp. latifolia (Cosson) Rivas Martínez, Deschampsia stricta, Helianthemum nummularium, Lotus glareosus var. villosus, Pedicularis sylvatica subsp. lusitanica, Prunus spinosa, Quercus x fontqueri y Quercus pyrenaica. El subpiso mesomediterráneo inferior se distingue por la ausencia de elementos termomediterráneos y la presencia de otras plantas sensibles a las heladas como Myrtus communis, Olea europaea var. sylvestris, Pistacia lentiscus, Phlomis purpurea, Rhododendron ponticum, y Rubia peregrina subsp. longifolia.

El efecto de la oceanidad es muy evidente no sólo en la franja costera, reflejándose en formaciones características como los brezales aljíbicos de influencia atlántica, la vegetación de tipo lauroide de las gargantas profundas que forman las areniscas del Aljibe, los pastizales vivaces de elevada biomasa y los herbazales escionitrófilos de lindero de los bosques.

En el territorio estudiado existen cuatro ombrotipos: seco, subhúmedo, húmedo e hiperhúmedo. De esta riqueza ómbrica es responsable el relieve y la apertura de las sierras más occidentales hacia los vientos húmedos de poniente. También las precipitaciones de carácter orográfico adquieren una gran importancia en estas sierras; las masas de aire del atlántico ascienden por las laderas y se enfrían por expansión, aumentando la humedad relativa y provocando la saturación y 
condensación, con nubosidad de tipo cumuliforme, y se llega a formar un bosque de nieblas. Naturalmente, lo que aleja esta situación de un bosque tropical es la xericidad estival, aunque ésta puede verse amortiguada por la formación de nieblas causadas por el viento de Levante (Rivas Goday, 1967). Estas situaciones meteorológicas van a permitir que determinadas plantas relictas del Terciario se refugien en las gargantas aljíbicas (Culcita macrocarpa, Davallia canariensis, Laurus nobilis y Rhododendron ponticum) formando comunidades en el interior de bosques caducifolios (alisedas y quejigales) y también la existencia de extensos y vigorosos alcornocales (Pérez Latorre et al., 1993; Nieto et al., 1994).

\section{B. Sectorización fitogeográfica}

Proponemos la siguiente sectorización fitogeográfica para el territorio estudiado (fig. 1):

Región Mediterránea

Subregión Mediterránea Occidental

Superprovincia Iberomarroquí-atlántica

Provincia Tingitano-Onubo-Algarviense Sector Aljíbico
1. Subsector Aljíbico
2. Subsector Algecireño
3. Subsector Marbellí
4. Subsector Sidonense

\section{Superprovincia Iberomarroquí-atlántica}

Son los territorios de la Península Ibérica y norte-oeste de Africa con un régimen ómbrico atlántico-mediterráneo (Gaussen et al., 1958), predominio de sustratos silíceos y que forman un arco alrededor del Golfo de Cádiz, idea que se aproxima a la gran unidad del SO mediterráneo de Takhtajan (1986). Según la lista de taxones que propone Quézel (1957) para enlazar los mundos eurosiberiano y mediterráneo, y la distribución de elementos atlánticos en el norte de Africa (Dahlgren y Lassen, 1972), esta superprovincia significa un espacio migratorio de especies atlánticas hacia el $\mathrm{S}$ de la Península Ibérica y $\mathrm{N}$ de Africa. Además, perviven en ésta zona elementos lauroides y pteridófitos relictos del terciario (Barbero et al., 1994; Pichi Sermoli et al., 1988, 1991) que enlazan ésta superprovincia con la región Macaronésica donde aún perviven éstas formaciones.

Algunas especies territoriales iberomarroquí-atlánticas presentes en el sector Aljíbico (* también en la Península Tingitana) son las siguientes: Agrostis castellana (*), A. pourretii (*), A. reuteri (*), Allium scorzonerifolium, Avenula sulcata subsp. albinervis (*), Biscutella baetica (*), Cistus populifolius subsp. major $(*)$, Coronilla dura (*), Cytisus baeticus (*), Delphinium pentagynum (*), Deschampsia stricta, Echinospartum algibicum, Erica australis (*), Euphorbia transtagana (*), Festuca ampla (*), Frangula alnus subsp. baetica (*), Galium viscosum (*), Genista hirsuta subsp. lanuginosa (*), Genista triacanthos (*), Genista tridentata (*), Halimium commutatum $\left.{ }^{*}\right)$, Halimium alyssoides subsp. lasianthum (*), Klasea monardii, Lavandula luisieri, Leucojum trichophyllum (*), Linaria viscosa, Lithodora prostrata subsp. lusitanica $(*)$, Lotus glareosus var. villosus, Luzula forsteri subsp. baetica (*), Misopates orontium var. grandiflorum (*), Myosotis welwitschii (*), Nothobartsia aspera (*), Odontites tenuifolia, Pedicularis sylvatica subsp. lusitanica (*), Polygala microphylla, Pistorinia brevifolia (*), Pyrus bourgaeana, Quercus broteroi (*), Quercus lusitanica (*), Ranunculus bulbosus subsp. adscendens, Reseda media (*), Rhododendron ponticum, Salvia barrelieri (*), Salvia sclareoides, Scabiosa simplex subsp. dentata (*), Scilla monophyllos (*), Scrophularia lyrata (*), S. sambucifolia $(*)$, Senecio lopezii, Silene scabriflora subsp. tuberculata, Stipa gigantea (*), Thymelaea villosa (*), Thymus zygis subsp. sylvestris, Trifolium isthmocarpum (*), Trifolium 
michelianum (*) y Trisetaria scabriuscula.

\section{Provincia Tingitano-Onubo-Algarviense}

La provincia Tingitano-OnuboAlgarviense comprende un amplio territorio que se extiende desde la desembocadura del río Vouga en Aveiro (Portugal) hasta los arenales del Bajo Loukkos (Marruecos), y se divide en ocho sectores (Rivas-Martínez et al., 1990; Galán de Mera y Vicente Orellana, 1996): Ribatagano-Sadense, Divisorio portugués, Monchiquense, Algarviense, GaditanoOnubense, Aljíbico, Tingitano y del Bajo Loukkos.

\section{Sector Aljíbico}

El sector Aljíbico destaca por su gran relación con el sector Tingitano (Ojeda et al., 1996), incluso en sus áreas más orientales (Marbella), donde la vegetación guarda una semejanza manifiesta con las zonas basales silíceas del Rif (Marruecos). Se extiende por el oeste desde el río Guadalete (Jerez de la Frontera, Cádiz) hasta Fuengirola y el Valle del Genal (Málaga) por el este. Por el norte, su límite es el curso del río Majaceite, coincidiendo con las últimas estribaciones de las areniscas siliceas y el comienzo de las calizas béticas (sierra de las Cabras), que albergan una vegetación de origen diferente. Aunque el límite sur del sector es el estrecho de Gibraltar, es una frontera no carente de dificultades puesto que hay taxones y sintaxones característicos comunes entre los sectores Aljíbico y Tingitano. Sin embargo, pensamos que la barrera del estrecho es 10 suficientemente grande como para considerar dos unidades diferentes. Aunque las formaciones vegetales sean semejantes, hay algunos sintaxones distintos y un grupo de especies que no atraviesan el estrecho en sentido norte (Deil in Refass, 1993) como Chamalepeuce abylense, Rhodanthemum hosmariense, R. laouense, Silene lagrangei, Sonchus fragilis, Stemmacantha longifolia var. ericeticola y Teucrium afrum subsp. rubriflorum.

Algunas especies aljíbicas endémicas (+) o presentes también en la Península Tingitana (*) son las siguientes:Armeria hirta (*), Asphodelus roseus (*), Brachypodium sylvaticum var. gaditanum (+), Bellis rotundifolia (*), Bupleurum foliosum (*), Carduus lusitanicus subsp. santacreui (+), Carex elata subsp. mauritanica (*), Crepis tingitana (*), Cytisus striatus subsp. welwitschii (+), C. tribracteolatus (+), Digitalis purpurea subsp. bocquetii (+), Echinospartum algibicum (+), Festuca baetica $(*), F$. boissieri (*), Holcus grandiflorus (+), Klasea alcalae $\left(^{*}\right)$, Leontodon tingitanus (*), Limonium emarginatum (*), Narcissus viridiflorus (*), Polygala baetica (*), Satureja salzmannii (*), Scrophularia laevigata (*), Sedum hirsutum subsp. baeticum (*), Silene gaditana (+), Teucrium scorodonia subsp. baeticum (*), Tolpis nemoralis (*) y Ulex borgiae (*).

El sector Aljíbico se divide en cuatro subsectores: Sidonense al oeste, Algecireño al sur, Aljíbico al norte y Marbellí al este (figura 1).

\section{Subsector Aljíbico}

Está limitado al sur por la sierra Blanquilla; al norte por el río Majaceite, la sierra de Grazalema y la sierra de las Cabras; al oeste por el río Barbate; y al este por la sierra Bermeja. Se caracteriza por un termoclima termomediterráneo superior y mesomediterráneo (inferior y medio) con una continentalidad que no permite la abundancia de elementos relictos. El uso del territorio es fundamentalmente forestal y ganadero.

Son especies diferenciales y endémicas (+): Asplenium sagittatum, Deschampsia stricta, Festuca baetica, Echinospartum algibicum (+), Helianthemum nummularium, Lotus glareosus var. villosus, Narcissus cantabricus, Prunus spinosa y Quercus $x$ fontqueri. 


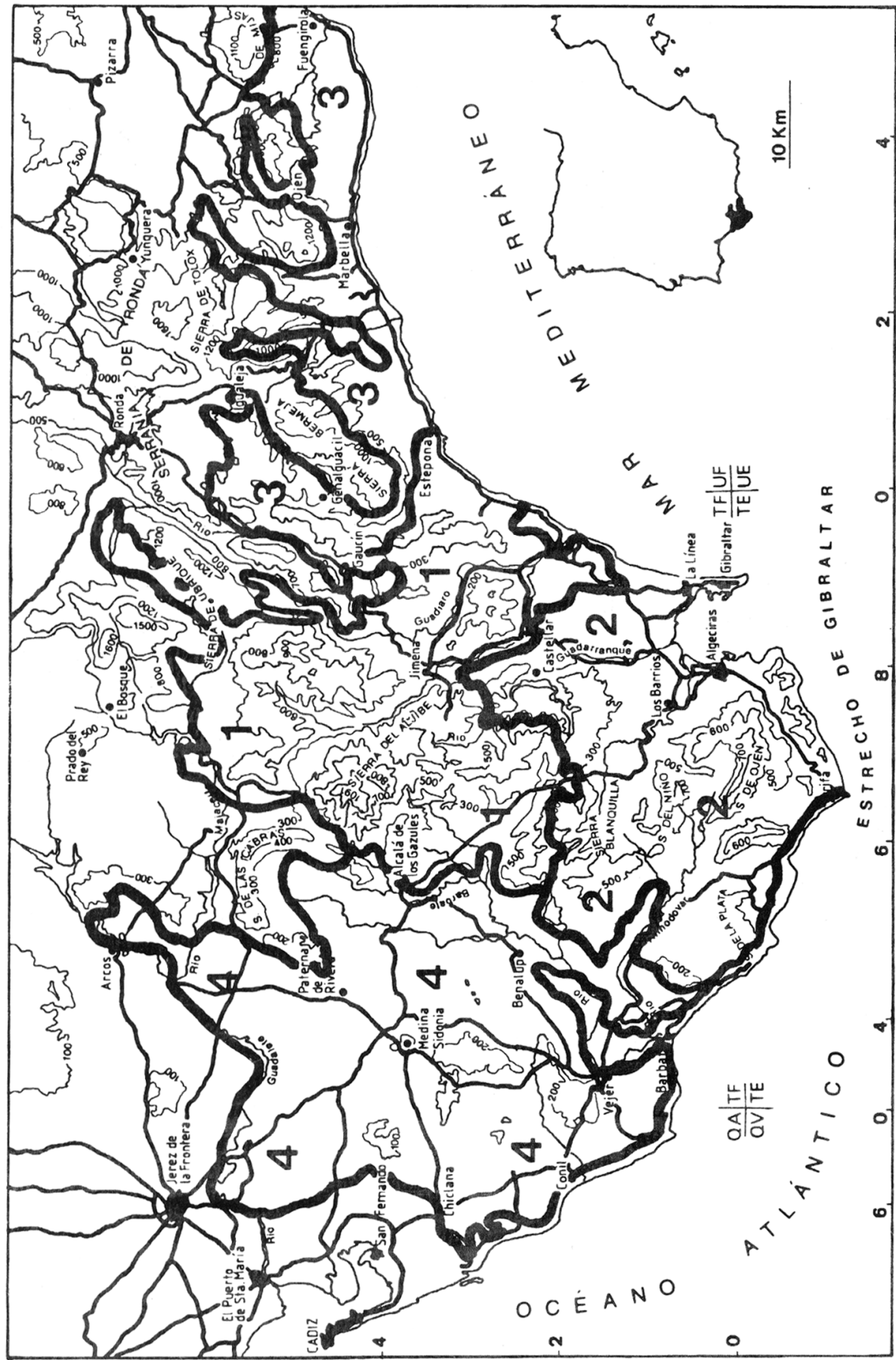

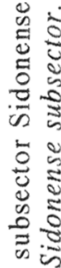

子ं

जิ

帘

$\sum$

웡

站

mंn

过

는

인

농

式

के

तं

8

绶

《告

5. क

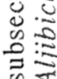

$\therefore$

$\ddot{8}$

:

流。

홍

क

สู

元

on:

o

든

등

3

$\therefore$ 


\section{Subsector Algecireño}

Comprende las áreas más térmicas (termomediterráneo inferior), húmedas y oceánicas del territorio, lo que permite el desarrollo de gran número de elementos paleomediterráneos y relictos tropicales (Galán de Mera et al., 1995c), abarcando también zonas termomediterráneo superiores y mesomediterráneas inferiores. Se extiende desde las sierras Blanquilla y del Niño hasta el estrecho de Gibraltar; su límite oriental es el valle del río Guadiaro. El uso del territorio es forestal y ganadero, con importantes zonas industriales concentradas en la Bahía de Algeciras. Son especies diferenciales y endémicas (+): Asphodelus roseus, Asplenium marinum, Carduus lusitanicus subsp. santacreui (+), Christella dentata, Cladanthus arabicus, Culcita macrocarpa, Cytisus striatus subsp. welwitschii (+), Daphne laureola subsp. latifolia, Diplazium caudatum, Dryopteris guanchica, Polypodium macaronesicum, Psilotum nudum, Pteris incompleta, Silene coarctata, Silene gaditana (+) y Vandenboschia speciosa.

Siguiendo la idea fitogeográfica de los territorios de superficie continua (BraunBlanquet, 1923; Meusel et al., 1965) incluimos al peñón de Gibraltar dentro del subsector Algecireño. El peñón de Gibraltar es de origen jurásico y tiene una gran semejanza con las alineaciones del Haouz (Marruecos). Su independencia fitogeográfica (a nivel de distrito) se mantiene en virtud de algún endemismo como Saxifraga globulifera var. gibraltarica, de plantas exclusivas dentro del sector como Ferula tingitana, Iberis gibraltarica, Lavatera arborea, L. maritima, Silene tomentosa, Succowia balearica y Thymus willdenowii) y de su vegetación (Deil, 1994; Linares et al., 1996).

\section{Subsector Marbelli}

El subsector Marbellí se extiende desde Estepona a Fuengirola por la costa y al valle del Genal por el interior, siguiendo la litología paleozoica de pizarras, cuarcitas, micaesquistos, gneises y paleodunas. El carácter oriental de esta parte del sector Aljíbico se caracteriza por menores precipitaciones y ombroclima predominantemente subhúmedo, lo que conduce a la ausencia de taxones propios de los subsectores Aljíbico y Algecireño y la presencia de otros más relacionados con las sierras béticas situadas al norte y este del subsector. El uso del territorio es turístico en el litoral y forestal en el interior. Son especies diferenciales las siguientes: Abies pinsapo, Coriaria myrtifolia, Doronicum plantagineum, Dryopteris affinis subsp. affinis, Genista hirsuta lanuginosa, Genista umbellata, Halimium atriplicifolium, Hypericum androsaemum, Quercus broteroi, Rupicapnos africana subsp. decipiens y Ulex baeticus.

\section{Subsector Sidonense}

Se extiende desde la margen izquierda del río Barbate hasta el río Guadalete, en las proximidades de Jerez de la Frontera. Es el más heterogéneo de todos los subsectores debido a su gran diversidad de sustratos. Destacan los afloramientos de areniscas silíceas y la abundancia de margas, arcillas y calcarenitas, que conducen a la presencia de un elevado número de elementos florísticos diferenciales. El uso del territorio es fundamentalmente agrícola y ganadero. Son especies diferenciales ( + endemismos): Anthemis bourgaei (+), Arenaria hispanica, Avena murphyi, Avenula sulcata subsp. gaditana, Bupleurum rigidum subsp. paniculatum, Chaetopogon fasciculatus, Euphorbia gaditana, Festuca arundinacea subsp. atlantigena, Fumana juniperina, Genista hirsuta subsp. hirsuta, Globularia alypum, Glossopappus macrotus, Hedysarum glomeratum, Klasea monardii, Lepidium subulatum, Margotia gummifera, Mercurialis elliptica, Ononis azcaratei, Parapholis 
incurva, P. pycnantha, Ranunculus graminifolium, Reseda stricta, Salvia tingitana, S. sclareoides, Scrophularia sambucifolia subsp. mellifera, Sideritis grandiflora, Silene stockeni (+), Tanacetum annuum, Trifolium pallidum, Trisetaria scabriuscula, Verbascum erosum, Vicia lutea subsp. cavanillesii y Ulex baeticus subsp. scaber (+).

\section{Series de vegetación}

Los bosques predominantes en todo el sector Aljíbico son los alcornocales, quejigales y acebuchales. Los primeros coronan el edificio de la vegetación climatófila (fig. 2), mientras que los acebuchales tienen un sentido edafófilo (Nieto Caldera et al., 1991).

1. Serie termomediterránea TingitanoOnubo-Algarviense, Luso-Extremadurense y Bética, subhúmeda y silicícola-sabulícola del alcornoque (Quercus suber). Myrto communis-Querceto suberis sigmetum.

Los alcornocales de Myrto communisQuercetum suberis ocupan las áreas basales y menos lluviosas de los sectores Aljíbico, Gaditano-Onubense, Algarviense, Monchiquense, Araceno-Pacense, Tingitano, Bajo Loukkos y grandes extensiones sobre sustratos silíceos compactos del Rif.

El manejo de estos bosques conduce a un madroñal deCytiso baetici-Arbutetum unedonis quercetosum cocciferae como orla o primera etapa de sustitución, acompañado a veces de pastizales de lindero (Calamintho-Galietum scabri). Sobre suelos muy empobrecidos el madroñal es reemplazado por un espinal de Asparago-Calicotometum villosae con características propias, y a que está enriquecido en algunos puntos de la costa con Juniperus turbinata, lo que nos lleva a describir una nueva subasociación: AsparagoCalicotometum villosae juniperetosum turbinatae subass.nova (Asparago-Rhamnion, Pistacio-Rhamnetalia, Quercetea ilicis); sintypus: CADIZ, Zahara de los Atunes, Faro del Camarinal, $100 \mathrm{~m}^{2}$. Pistacia lentiscus 4, Calicotome villosa 1, Chamaerops humilis 2, Phlomis purpurea 1, Juniperus turbinata 2,

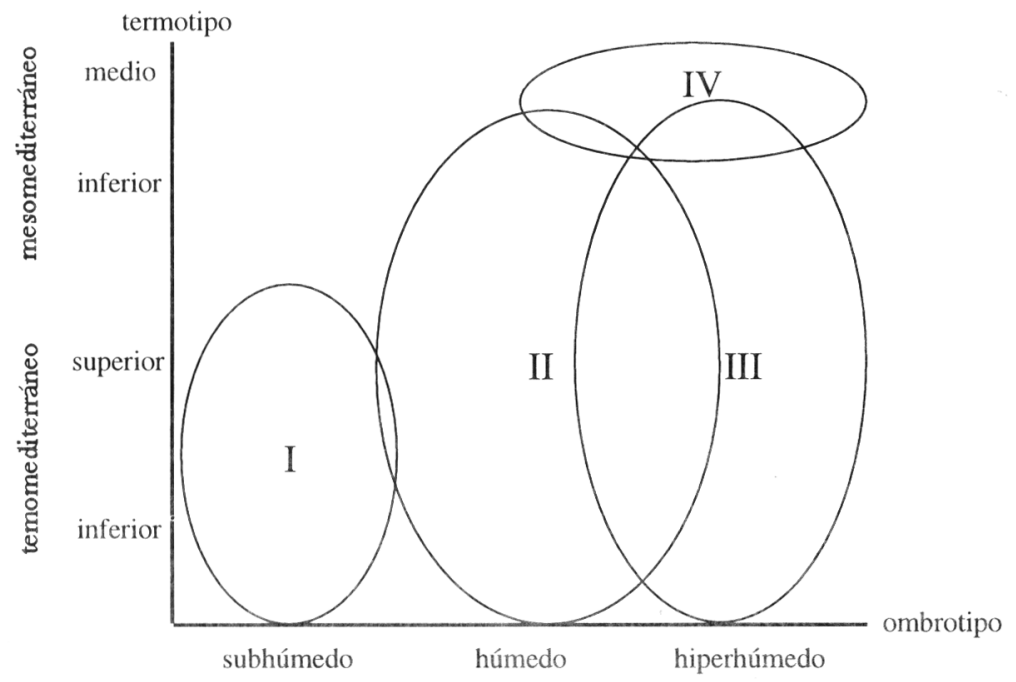

Fig. 2. Posición ecológica de las series de vegetación climatófilas respecto a termotipo y ombrotipo (índices bioclimáticos).I: Myrto-Querceto suberis sigmetum, II: Teucrio-Querceto suberis sigmetum, III: RuscoQuerceto suberis sigmetum, IV: Cytiso-Querceto pyrenaicaesigmetum. Ecological behaviour of the climatophyll vegetation series in relation to bioclimatic indexes (termotype and ombrotype). 
Quercus coccifera 1, Teucrium fruticans 1, Olea europaea var. sylvestris 1, Smilax aspera 3, Phillyrea angustifolia 1, Ruscus aculeatus 1, Rubia peregrina 1, Brachypodium retusum 1, Asphodelus ramosus 1, Hyparrhenia podotricha 1, Cistus crispus + , Lavandula stoechas + , Cistus ladanifer + .

Los jarales de sustitución sobre gneises, micaesquistos y pizarras pertenecen al Calicotomo-Genistetum lanuginosae (en parte del subsector Marbellí occidental) y al Calicotomo-Genistetum lanuginosae genistetosum umbellatae (en el subsector Marbellí oriental); sobre areniscas estos jarales se incluyen en la subasociación CalicotomoGenistetum lanuginosae genistetosum triacanthi (subsectores Aljíbico, Algecireño y Sidonense). La etapa de pastizal es muy variada según la influencia antrópica, aunque son destacables las asociaciones Trifolio cherleriPlantaginetum bellardii y Trifolio pallidiVulpietum geniculatae.

En las zonas arenosas próximas a la costa gaditana (Conil de la Frontera, Chiclana de la Frontera y Sotogrande), en el interior de la provincia (San José del Valle y Arcos de la Frontera) y en la costa malagueña (entre Calahonda y Marbella), la faciación típica de estos alcornocales es sustituida por una subserie sobre arenas (Myrto communis-Querceto suberis halimietoso sigmetum). La etapa arbustiva en puntos próximos a la costa es un espinal de Asparago-Rhamnetum oleoidis ericetosum scoparii con Juniperus turbinata, mientras que el matorral bajo pertenece a asociaciones de la alianza Coremion albi (Cisto-Ulicetum australis) y el pastizal a Malcomio-Vulpietum alopecuris. En la provincia de Cádiz, entre las poblaciones de Conil y Chiclana son abundantes los conglomerados en cuyos suelos superficiales desaparece Stauracanthus genistoides instalándose entonces la asociación FumanoCistetum crispi. El pastizal más importante en esta faciación, fuera de la influencia marítima, es la comunidad terofítica de Anthyllido hamosae-Malcolmietum lacerae.

2. Serie termo-mesomediterránea aljíbica, bética y tigitana, húmedo-hiperhúmeda del alcornoque (Quercus suber). Teucrio baeticiQuerceto suberis sigmetum.

Son los alcornocales (Teucrio baeticiQuercetum suberis) de las áreas más húmedas del sector Aljíbico, alcanzando algunos territorios en la Península Tingitana. Ocupan una franja entre los 200 y $900 \mathrm{~m}$. dentro de los subsectores Aljíbico, Algecireño y áreas más umbrías del subsector Marbellí. La limpieza tradicional del monte, para favorecer la extracción del corcho, conduce a una formación con madroños y genisteas de Cytiso baeticiArbutetum unedonis y a comunidades vivaces de Calamintho-Galietum scabri digitaletosum bocquetii en los subsectores Aljíbico, Algecireño y parte del Marbellí (valle del Genal) y lamietosum flexuosi en los alcornocales con Quercus canariensis. Al degradarse el madroñal se origina un espinal de Asparago aphylli-Calicotometum villosae genistetosum linifoliae rico en genisteas ombrófilas (Cytisus villosus, Genista linifolia y Genista monspessulana). El sobrepastoreo, rozas e incendios recurrentes erosionan el suelo, entrando en juego procesos de podsolización y permitiendo la instalación de un brezal-ahulagar (Genisto tridentisStauracanthetum boivinii). En los suelos más profundos y humificados de este brezal se instala la subasociación quercetosum lusitanicae, que se comporta como comunidad permanente de crestas y lugares expuestos a los fuertes vientos, donde no se puede desarrollar la vegetación arbórea (Ceballos et al., 1933). La subasociación ulicetosum baetici de estos brezales, de areal Marbellí, se desarrolla sobre sustratos esquistosos y marca la disminución de las precipitaciones hacia oriente (Pérez Latorre et al., 1993). El brezal de Genisto-Stauracanthetum aparece como 
Tabla 1

Comunidad de Juniperus oxycedrus (Pistacio-Rhamnetalia, Quercetea ilicis)

\begin{tabular}{lccr}
\hline Altitud & 250 & 250 & 300 \\
Cobertura & 90 & 100 & 50 \\
Area & 200 & 150 & 100 \\
Inclinación & 15 & 25 & 30 \\
Orientación & SW & SE & SE \\
Número de especies & 26 & 18 & 16 \\
Número de orden & 1 & 2 & 3
\end{tabular}

Comunidad

Juniperus oxycedrus

Osyris quadripartita

Cytisus tribracteolatus

Adenocarpus telonensis

Características de Pistacio-Rhamnetalia

Phillyrea angustifolia

Arbutus unedo

Myrtus communis

Erica arborea

Viburnum tinus

$\begin{array}{lll}4 & 4 & 2 \\ + & 1 & 3 \\ + & + & . \\ 1 & 1 & . \\ + & . & .\end{array}$

Características de Quercetea

Quercus suber

Daphne gnidium

Olea europaea

Rubia peregrina

Ruscus aculeatus

Ruscus hypophyllum

Smilax aspera var. altissima

Características territoriales

Stauracanthus boivinii

Teucrium baeticum

Nothobartsia aspera

Compañeras

Calicotome villosa

Calluna vulgaris

Cistus ladanifer

Erica australis

Lavandula stoechas

Erica scoparia

Asphodelus albus

Cistus salvifolius

Erica umbellata

Romulea bulbocodium

Localidades: 1, 2 y 3.- El Salado, Jimena de la Frontera, Cádiz. vegetación permanente en las cumbres del macizo del Aljibe, en situaciones de suelos poco desarrollados. En exposiciones elevadas con suelo arenoso, el brezal-ahulagar es sustituído por un jaral-brezal con Erica australis (Nezadal et al., 1994) en cuya composición destacan Genista tridentata y Cistus populifolius subsp. major. En áreas de suelos poco evolucionados, los claros del brezal-ahulagar están ocupados por un pastizal xerófilo con Deschampsia stricta y Agrostis curtisii. En el sector Aljíbico encontramos la asociación Stauracantho boiviniiDrosophylletum lusitanici saturejetosum salzmannii, muy próxima al GenistoStauracanthetum, propia de taludes y suelos degradados por la acción del fuego (Galán de Mera y Vicente Orellana, 1996) y que se desarrolla tanto sobre areniscas como sobre esquistos.

En zonas puntuales del subsector Algecireño, con frecuentes nieblas y altitudes por encima de $650 \mathrm{~m}$ aparece una comunidad de quejigueta (Quercus lusitanica) con acebos (Ilex aquifolium) y quejigos (Quercus canariensis), sirviendo como testigo de la misma el siguiente inventario: CADIZ, Tarifa, Tajos del Espino. $150 \mathrm{~m}^{2}$. Areniscas. $725 \mathrm{~m}$. Cobertura $100 \%$. Orientación E. Quercus lusitanica 5, Ilex aquifolium 2, Quercus canariensis 1, Lonicera hispanica + , Hedera helix + , Tamus communis + , Smilax mauritanica + , Crataegus monogyna + , Arbutus unedo + , Daphne latifolia+, Pteridium aquilinum 1, Cistus major 1, Rubus ulmifolius 1, Pedicularis lusitanica +, Teucrium baeticum 1, Erica australis 1, Cistus salvifolius +, Asplenium onopteris + , Senecio lopezii + , Brachypodium gaditanum +, Erica arborea + , Digitalis bocquetti + .

En algunos puntos del subsector Aljíbico, a baja altitud, sobre litosuelos muy soleados y expuestos, se desarrolla una comunidad abierta con carácter relictual caracterizada por Juniperus oxycedrus (tabla 1). 
Los pastizales mejor representados dentro de esta serie de vegetación son los vallicares de Gaudinia fragilis y Agrostis castellana. Se encuentran también muy extendidos los pastizales nanoterofíticos de TrifolioPlantaginetum bellardii.

En función de determinadas condiciones ecológicas, estos alcornocales dan lugar a bosques mixtos con encinas, quejigos, robles y pinsapos. La subasociación quercetosum canariensis destaca en las vertientes orientales del macizo del Aljibe, en vaguadas y sobre suelos que muestran compensación edáfica (Díez Garretas et al., 1986; Pérez Latorre et al., 1994); los pastizales de lindero de este bosque son incluíbles en la subasociación Calamintho-Galietum lamietosum flexuosi. La subasociación quercetosum rotundifoliae fue descrita por Nieto Caldera et al. (1990) en la provincia de Málaga (subsector Marbellí) como un aspecto del alcornocal que tiende a ocupar situaciones continentalizadas y menos húmedas, o bien con suelos básicos. La variante de Quercus pyrenaica aparece sobre los $700 \mathrm{~m}$ de altitud en las sierras del Aljibe y de Algeciras. La variante de Abies pinsapo se encuentra en los ambientes más húmedos y umbríos del subsector Marbellí.

\section{Serie termo-mesomediterránea aljíbica} y tingitana, hiperhúmeda-húmeda silicícola del quejigo africano (Quercus canariensis). Rusco hypophylli-Querceto canariensis sigmetum.

Estos bosques de quejigo moruno, Rusco hypophylli-Quercetum canariensis, considerados como relícticos, son de óptimo termo-mesomediterráneo con ombroclima hiperhúmedo-húmedo y se sitúan preferentemente en zonas microclimáticamente favorables como valles con abundancia de criptoprecipitaciones o suelos con hidromorfía temporal en áreas menos lluviosas. En el subsector Aljíbico estos quejigales presentan las mismas etapas de sustitución que los alcornocales de Teucrio-Quercetum suberis quercetosum canariensis. En el subsector Algecireño, más húmedo, el bosque se enriquece en elementos de hoja lauroide (Daphne laureola subsp. latifolia, Ilex aquifolium, Laurus nobilis, Viburnum tinus, etc.) constituyendo la subasociación lauretosum nobilis (Nezadal et al., 1994) y su etapa de sustitución es un zarzal de Lonicero hispanicae-Rubetum ulmifolii, al igual que sucede en el contacto con las formaciones de hojaranzos, Rusco-Quercetum canariensis rhododendretosum baetici. En el subsector Marbellí el enriquecimiento del quejigal con Quercus broteroi y Quercus x marianica nos permite describir una nueva subasociación Rusco-Quercetum canariensis quercetosum broteroi subass. nova (tabla 2, typus inv. 4).

\section{Serie mesomediterránea aljíbica y} rifeña, húmedo-hiperhúmeda silicícola del roble melojo (Quercus pyrenaica). Cytiso triflori-Querceto pyrenaicae sigmetum.

Melojares ombrófilos (Cytiso trifloriQuercetum pyrenaicae), que tienen las mejores representaciones en las proximidades de las cumbres del Aljibe y puntualmente en las Sierras de Algeciras (Cádiz), Valle del Genal, Sierra de Ronda y Montes de Cortes (Málaga). Aparecen expuestos a los vientos atlánticos y, en general, protegidos del viento de levante. Ceballos y Martín Bolaños (1930), Rivas Goday et al. (in González García, 1965) y RivasMartínez (1987) advirtieron sobre la existencia de estas formaciones de Quercus pyrenaica en la cumbre del Aljibe, incluyéndolos en el seno de los melojares luso-extremadurenses (ArbutoQuercetum pyrenaicae) y tratándolos como una disyunción. Sin embargo, al ser los melojares más meridionales de la Península Ibérica y muy próximos geográficamente y florísticamente a los norteafricanos, con los que comparten especies características (Agrostis curtisii, Avenula sulcata subsp. albinervis, Clinopodium vulgare subsp. 
Tabla 2

Rusco hypophylli-Quercetum canariensis quercetosum broteroi subass. nova (Quercetea ilicis, Quercetalia,Querco-Oleion)

\begin{tabular}{lccccc}
\hline Inclinación $\left({ }^{\circ}\right)$ & 30 & 20 & 60 & 15 & 45 \\
Cobertura $(\%)$ & 100 & 100 & 90 & 100 & 100 \\
Exposición & $\mathrm{N}$ & $\mathrm{O}$ & $\mathrm{N}$ & $\mathrm{N}$ & $\mathrm{NE}$ \\
Altitud $(1=10 \mathrm{~m})$. & 44 & 50 & 60 & 53 & 55 \\
Area $(\mathrm{m} 2)$ & 100 & 100 & 400 & 200 & 100 \\
Número de especies & 1 & 2 & 3 & 4 & 5 \\
$\mathrm{~N}^{\mathrm{o}}$ de orden & & &
\end{tabular}

Características de asociación y subasociación

Quercus faginea broteroi

Quercus x marianica

Quercus canariensis

Polystichum setiferum

Polypodium cambricum

Davallia canariensis

Características de Pistacio-Rhamnetalia

Erica arborea

Genista monspessulana

Cytisus baeticus

Teucrium fruticans

Cytisus villosus

Phillyrea latifolia

Crataegus brevispina

Genista linifolia

Características de Quercetea

Rubia peregrina

Asplenium onopteris

Quercus suber

Smilax aspera

Carex distachya

Melica arrecta

Smilax aspera var. altissima

Hedera helix

Rubia agostinhoi

Ruscus aculeatus

Compañeras

Pteridium aquilinum

Digitalis purpurea bocquetti

Tamus communis

Rubus ulmifolius

Vinca difformis

Galium scabrum

Calamintha baetica

Digitalis tomentosa

Brachypodium sylvaticum

Hypericum androsaemum

Daphne gnidium

Bellis perennis

Origanum virens

Luzula forsteri baetica

$\begin{array}{lllll}1 & 2 & 2 & 3 & 1 \\ 1 & 1 & 1 & 2 & 3 \\ 2 & 1 & 1 & 1 & 1 \\ 2 & + & + & 1 & 1 \\ + & . & + & . & . \\ . & . & + & . & .\end{array}$

Localidades: 1, 2, 3, 4.- Monte del Duque, Casares. Málaga. 5.- El Daidín, Benahavís. Málaga. 
Tabla 3

Cytiso triflori-Quercetum pyrenaicae

(Querco-Fagetea, Quercion robori-pyrenaicae,Quercenion pyrenaicae)

Area (m2)

Exposición

Altitud $(1=10 \mathrm{~m}$.

Número de orden

Características

Quercus pyrenaica

Luzula forsteri baetica

Avenula albinervis

Genista monspessulana

Agrostis curtisii

Quercus canariensis

Cytisus villosus

Senecio lopezii

Quercus x fontqueri

Bellis rotundifolia

Características de Querco-Fagetea

Rubus ulmifolius

Ranunculus ficaria

Ilex aquifolium

Prunus spinosa

Allium scorzonerifolium

Crataegus monogyna brevispina

Características de Quercetea ilicis

Arbutus unedo

Daphne gnidium

Erica arborea

Quercus suber

Carex distachya

Smilax aspera

Arenaria montana

Viburnum tinus

Carex depressa

Quercus lusitanica

Compañeras

Cistus salvifolius

Erica australis

Pteridium aquilinum

Cistus populifolius major

Ulex borgiae

Dactylis glomerata hispanica

Holcus lanatus

Holcus mollis

Brachypodium gaditanum

Lavandula stoechas

Cistus crispus

Calluna vulgaris

Erica scoparia

Genista tridens

Stauracanthus boivinii

Halimium alyssoides lasianthum

Teucrium scorodonia baeticum

$\begin{array}{ccccccccc}80 & 50 & 50 & 100 & 100 & 75 & 100 & 100 & - \\ \mathrm{NO} & \mathrm{N} & \mathrm{N} & \mathrm{N} & \mathrm{O} & - & \mathrm{O} & \mathrm{S} & - \\ 100 & 85 & 100 & 100 & 100 & 76 & 130 & 120 & - \\ 1 & 2 & 3 & 4 & 5 & 6 & 7 & 8 & 9\end{array}$

$\begin{array}{ccccccccc}3 & 3 & 4 & 4 & 4 & 4 & 5 & 5 & \text { V } \\ 1 & . & 1 & 1 & 1 & + & + & + & \text { V } \\ . & + & + & . & 1 & + & 1 & + & \text { III } \\ . & . & . & . & . & . & + & + & \text { III } \\ . & . & + & . & . & . & + & . & \text { II } \\ + & . & . & . & . & . & . & . & \text { I } \\ . & . & . & . & 1 & . & . & . & \text { V } \\ . & . & . & + & 1 & . & . & . & . \\ + & . & . & . & . & + & . & . & . \\ . & . & . & . & . & . & + & . & .\end{array}$

III

III

III

II

IV

Localidades: 1.- Casa de la Moracha, Alcalá de los Gazules, Cádiz. 2 y 5.- Garganta de Puerto Oscuro, Alcalá de los Gazules , Cádiz. 3.- entre el Aljibe y el Gurugú, Alcalá de los Gazules Cádiz. 6.- Tajos de la Corza, Tarifa, Cádiz. 7 y 8.- Jbel Alam, Larache, Marruecos. 9.- Barbero et al., 1981, tabla 31. 
Tabla 4

Asperulo hirsutae-Ulicetum scabri ulicetosum scabri

anthyllidetosum cytisoidis subass. nova.

(Rosmarinetea, Rosmarinetalia, Eryngio-Ulicion)

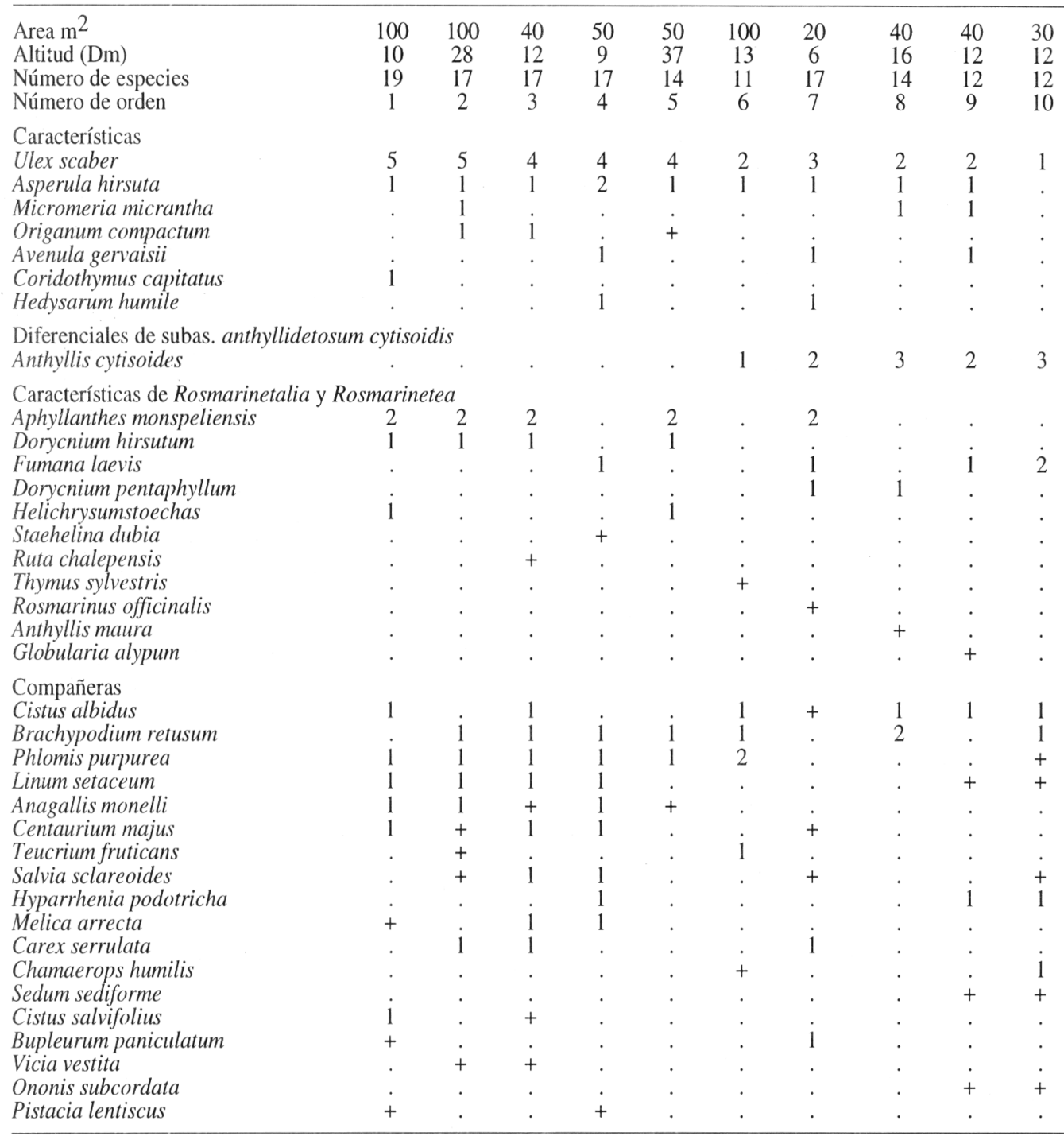

Otros taxones: Rubia longifolia + , Osyris alba + , Rhamnus oleoides + en 1; Campanula rapunculus + , Vinca difformis + , Smilax aspera,+ Quercus coccifera + , Carex distachya + en $2 ;$ Pulicaria odora + , Dactylis hispanica 1 en 3 ; Eryngium dilatatum + en 4; Lonicera implexa + , Osyris alba + , Daphne gnidium + , Scorzonera baetica + en 5; Onobrychis peduncularis + , Cistus monspeliensis 1 en 6; Picris comosa + , Astragalus echinatus 1, Calicotome villosa + en 8, Olea sylvestris + en 10 .

Localidades.- 1: Cabeza de Oveja, Jerez de la Frontera, Cádiz. 2: Montifarti, Jerez de la Frontera, Cádiz. 3: Tempul, Jerez de la Frontera, Cádiz. 4: Dehesa del Picado, Jerez de la Frontera, Cádiz. 5: Casa Hediondal, Jerez de la Frontera, Cádiz. 6: Venta de Tablada, Alcalá de los Gazules, Cádiz. 7: Entre el Mimbral y los Cuquillos, Jerez de la Frontera, Cádiz. 8: Carretera del Picacho, Alcalá de los Gazules, Cádiz. 9: Valdegama, Alcalá de los Gazules, Cádiz. 10: Cerro del Piojo, Alcalá de los Gazules, Cádiz. 
arundanum, Cytisus villosus, Festuca baetica, Genista monspessulana, Luzula forsteri subsp. baetica y Quercus canariensis), optamos por incluír estas formaciones en la asociación de los melojares rifeños (tabla 3).

Las etapas de sustitución en general coinciden con las del Teucrio-Quercetum suberis (brezales y quejigales enanos de Quercus lusitanica) aunque en áreas con compensación edáfica es sustituído por zarzales de Lonicero hispanicae-Rubetum ulmifolii. El pastizal más característico es la comunidad de Deschampsia stricta y Agrostis curtisii.

\section{Serie edafófila termo-} mesomediterránea, aljíbica, tingitana y atlásico-rifeña, subhúmedo-húmeda verticícola del acebuche (Olea europaea var. sylvestris). Tamo communis-Oleeto sylvestris sigmetum.

[Tamo communis-Oleetum sylvestris Benabid 1984 (Trav. Inst. Sci. Chérifien, Sér. Bot. 34: 1-64); lectotypus: tabla 2, inv. 26 (designado aquí)]

Los acebuchales de Tamo-Oleetum, frecuentes en el subsector Sidonense, son bosques que se desarrollan en áreas con vertisoles en las llanuras y cambisoles vérticos en las colinas margosas (bugeos), donde no pueden instalarse ni Quercus rotundifolia ni Quercus suber. Los cultivos y la presión del ganado vacuno originan una primera etapa de sustitución dominada por lentiscos (Asparago albi-Rhamnetum oleoidis). Al desaparecer el estrato edáfico de humus y aflorar las arcillas se instala el ahulagar de Asperulo hirsutaeUlicetum scabri que en lugares con afloramientos de materiales yesíferos se enriquece con Anthyllis cytisoides, por lo que proponemos para ésta situación ecológica la subasociación anthyllidetosum cytisoides subass. nova (tabla 4, typus inv. 7). Los pastizales vivaces característicos de las tierras de bugeos se incluyen en las comunidades de Phalaris coerulescens y Hedysarum coronarium.

En el distrito Gibraltareño, sobre materiales calizos, distinguimos una variante más húmeda de estos acebuchales caracterizada por Pistacia terebinthus.

En áreas con margas silíceas o margoareniscas se desarrolla la subasociación quercetosum suberis, cuyas etapas de sustitución corresponden al espinal de Asparago-Calicotometum villosae y a un jaral empobrecido incluíble en CalicotomoGenistetum lanuginosae genistetosum triacanthi .

6. Serie edafoxerófila termomediterránea, bética, aljíbica y mauritánica, subhúmedohúmeda del algarrobo (Ceratonia siliqua). Clematido cirrhosae-Ceratonieto siliquae sigmetum.

Los algarrobales de ClematidoCeratonietum siliquae (incl. Asparago albiRhamnetum oleoidis ceratonietosum siliquae Rivas Goday et al. 1965, n.n.) aparecen sobre calizas compactas, lapiaces y suelos poco evolucionados de los subsectores Aljíbico, Algecireño y Marbellí. Descrita por Barbéro et al. (1981) en el Rif, Medio Atlas y Alto Atlas (Marruecos), en nuestro territorio reconocemos a la faciación bética y aljíbica (phlomidetosum purpureae) de estos algarrobales.

La degradación de estos algarrobales conduce a un tomillar de Teucrio lusitaniciCoridothymetum capitati, y los pastizales más importantes son incluíbles en el orden Trachynietalia distachyae (Velezio rigidaeAstericetum aquaticae). En lapiaces y zonas karstificadas se desarrolla el pastizal crasifolio de Sedetum micrantho-sediformis.

\section{Las series riparias}

Están representadas por bosques edafohigrófilos en cauces permanentes (Querco-Fagetea) o formaciones arbustivas en cauces con grandes oscilaciones de caudal (Nerio-Tamaricetea). La degradación de los 
primeros conduce a los zarzales de Lonicero hispanicae-Rubetum ulmifolii o RuboCoriarietum myrtifolii (subsector Marbellí), mientras que las comunidades de NerioTamaricetea presentan etapas subseriales de juncales (Holoschoenetalia) o de vegetación de saladares y salinas (Sarlicornietea fruticosae, Thero-Salicornietea).

7a. Serie edafohigrófila aljíbica del hojaranzo (Rhododendron ponticum subsp. baeticum). Frangulo baeticae-Rhododendreto baetici sigmetum
En las cabeceras de las gargantas y también en algunas cumbres (sierras del Niño, Luna y Ojén) de los subsectores Aljíbico y Algecireño se instala una comunidad relíctica, Frangulo-Rhododendretum baetici, donde Rhododendron ponticum y otras especies lauroides (Laurus nobilis) se hacen dominantes. En el subsector Algecireño la asociación se enriquece con algunos elementos tropicales relictos (Pichi Sermolli et al., 1988) que nos permiten describir una variante con Culcita macrocarpa (tabla 5), facies termomediterránea inferior de lugares sometidos con mayor

\section{Tabla 5}

Frangulo baeticae-Rhododendretum baetici

var. de Culcita macrocarpa

(Querco-Fagetea, Populetalia, Osmundo-Alnion, Rhododendro-Alnenion)

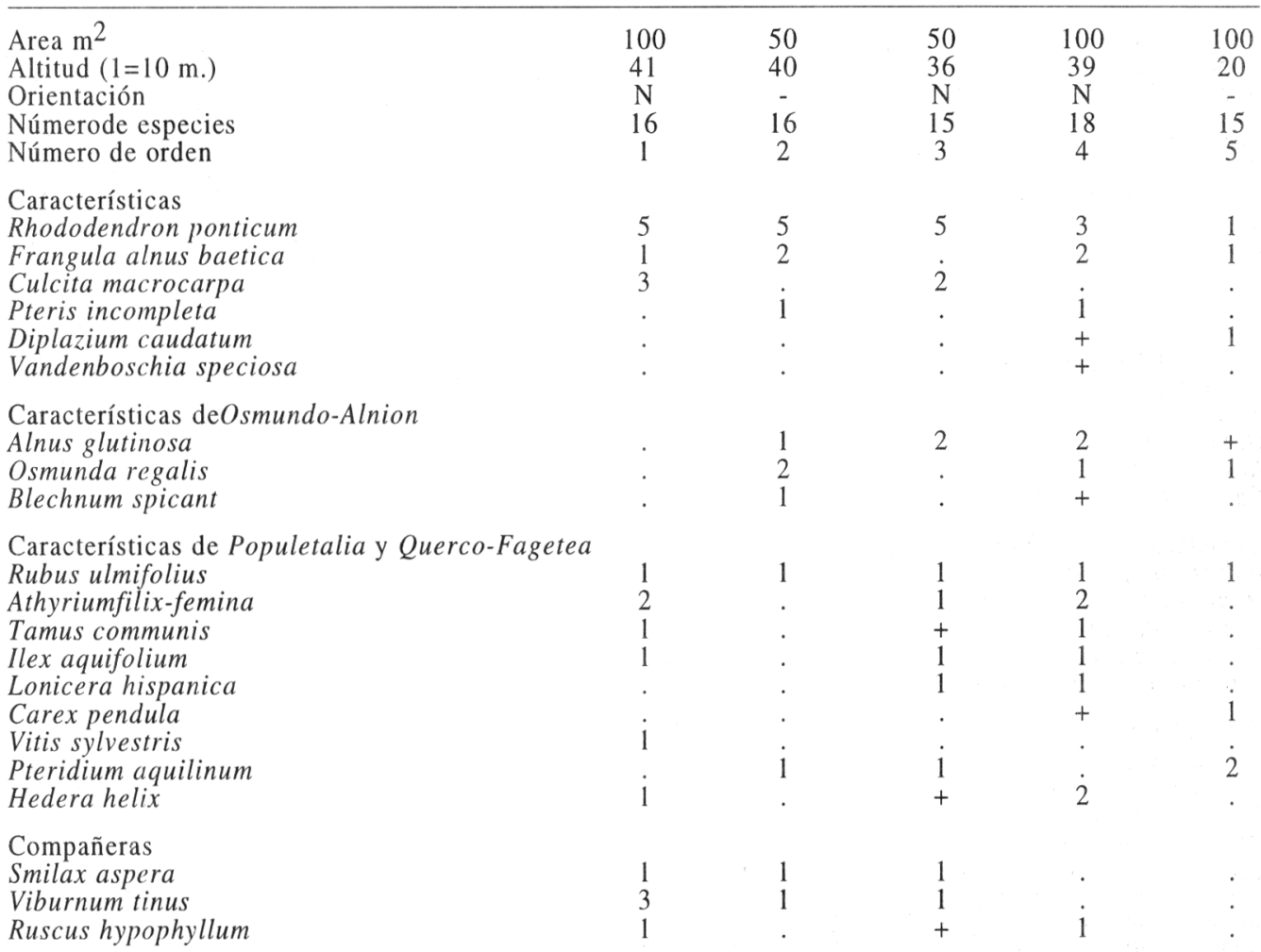

Otros taxones: Ruscus aculeatus 1 , Laurus nobilis 1 en $1 ;$ Erica arborea + , Digitalis bocquetii + en $2 ;$ Daphne gnidium 1 en 3; Quercus canariensis 2, Davallia canariensis 1 en 4; Carex lusitanica 1, Lobelia urens + , Oenanthe crocata 1 , Brachypodium gaditanum + , Nerium oleander + , Mercurialis reverchonii + , Gnaphalium luteo-album + en 5 .

Localidades.- 1 a 3: Dehesa de Ojén, Los Barrios, Cádiz. 4: Garganta del Gandelar, Sierra de Ojén, Los Barrios, Cádiz.

5: Molino de la Canaleja, Sierra de Enmedio, Tarifa, Cádiz. 
frecuencia a las nieblas causadas por los vientos atlánticos y de levante. La presencia de Nerium oleander en zonas más térmicas determina la existencia de la subasociación nerietosum oleandri.

7b. Serie edafohigrófila aljíbica del aliso (Alnus glutinosa). Arisaro proboscidei-Alneto glutinosae sigmetum

En las zonas bajas de las gargantas que llevan aguas tranquilas, las comunidades con hojaranzos son sustituídas por alisedas del Arisaro proboscidei-Alnetum glutinosae
(Ceballos y Martín Bolaños, 1930; Rivas Goday, 1968; Martínez Parras et al., 1987). Estos bosques riparios se caracterizan por la presencia de helechos (Athyrium filix-femina, Blechnum spicant y Osmunda regalis) de distinto significado biogeográfico que los del hojaranzal. Entre los grandes bloques silíceos de los cauces, las alisedas albergan una comunidad de cárices (Caricetum mauritanicae) de areal aljíbico y tingitano. En las partes bajas y más abiertas, con mayor fluctuación del cauce, reconocemos a la subasociación nerietosum oleandri.

Tabla 6

Crataego brevispinae-Populetum albae ass. nova (Querco-Fagetea, Populetalia, Populion, Fraxino-Ulmenion)

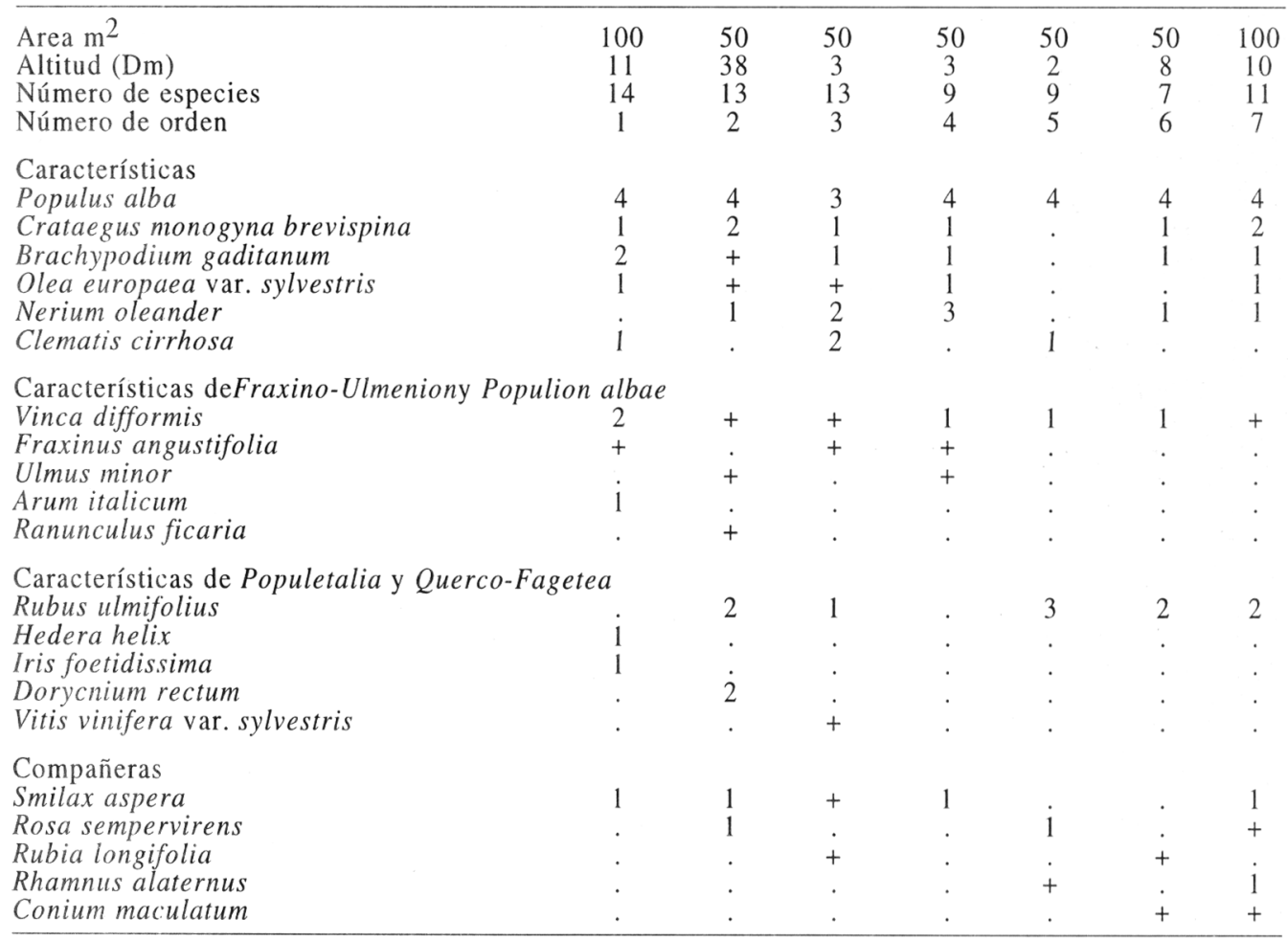

Otros taxones: Clematis flammula + , Smyrnium olusatrum + , Cirsium scabrum + en $1 ;$ Myrtus communis + , Rumex conglomeratus,+ Galega officinalis + en 2; Viburnum tinus + , Carex divulsa + en 3; Asparagus aphyllus + en 4; Galium aparine +, Aristolochia baetica +, Asparagus acutifolius + en 5; Urtica membranacea + en 6.

Localidades.- 1: Río Majaceite en la dehesa del Picado, Jerez de la Frontera, Cádiz. 2: El Sauzal, Alcalá de los Gazules, Cádiz. 3 y 4: río Rocinejo, Alcalá de los Gazules, Cádiz. 5: venta de la Rambla, Alcalá de los Gązules, Cádiz. 6: ventorrillo de Carrasco, Alcalá de los Gazules, Cádiz. 7: río Hozgarganta, Jimena de la Frontera, Cádiz. 
7c. Serie edafohigrófila aljíbica, onubense y algarviense del chopo (Populus alba). Crataego brevispinae-Populeto albae sigmetum

[Cabecera de serie: Crataego brevispinaePopuletum albae Galán de Mera ass. nova, tabla 6, typus inv. 7; incl. Nerio oleandriPopuletum albae Rivas Goday, F. Galiano y Rivas-Martínez 1965, n.n.]

Chopera de chopos blancos (Populus alba), termomediterránea, que puebla pequeñas depresiones arcillosas con suelos gleyzados de vega que pueden estar sometidos durante un escaso período de tiempo a inundaciones periódicas (Crataego-Populetum albae). Se presenta como un bosque rico en zarzas, así como en elementos de Quercetea ilicis que le dan un aspecto más xérico respecto a otras asociaciones con chopos (Irido-Populetum albae Tchou 1949, Rubio-Populetum albae BrB1. y O. Bolós 1957, Vinco-Populetum albae O. Bolós y Molinier 1958) y que en ocasiones alterna con las fresnedas. Son diferenciales frente al resto de las choperas ibéricas: Brachypodium sylvaticum var. gaditanum, Clematis cirrhosa, Crataegus monogyna subsp. brevispina, Nerium oleander y Olea europaea var. sylvestris. Es una asociación aljíbica, onubense y algarviense. Las choperas del norte de Argelia muestran una estructura y composición florística muy similar con una gran cantidad de elementos de Quercetea ilicis (Wojterski, 1985), aunque éstas han sido incluídas en el Irido-Populetum Tchou 1949.

7d. Serie edafohigrófila aljíbica y bética del sauce pedicelado (Salix pedicellata). Equiseto telmateiae-Saliceto pedicellatae sigmetum

Formación riparia de porte arbustivo, Equiseto-Salicetum pedicellatae, dominada por Salix pedicellata, que coloniza el lecho menor de los ríos intermitentes alternando en muchas ocasiones con el hojaranzal, pero ocupando biotopos más secos que éstos. En el valle del Genal (subsector Marbellí), con cauces más anchos y constantes, la comunidad se enriquece con sauces blancos (Salix alba) lo que permite describir la subasociación Equiseto-Salicetum pedicellatae salicetosum albae subass. nova (Salicion salvifoliae, Salicetalia purpureae, Querco-Fagetea); typus: MALAGA, Faraján, Río Genal, $200 \mathrm{~m}^{2}$. Limos y gravas. Salix alba 5, Equisetum telmateia 3, Rubus ulmifolius 3, Mentha suaveolens 1, Galium aparine 1, Salix pedicellata + , Scirpus holoschoeus + , Arum italicum +, Brachypodium sylvaticum +, Populus nigra + , Hedera helix + , Teucrium baeticum + , Dorycnium rectum + , Scrophularia scorodonia + , Vinca difformis + .

7e. Serie edafohigrófila iberomarroquíatlántica del fresno (Fraxinus angustifolia). Ranunculo ficariae-Fraxineto angustifoliae sigmetum

Las fresnedas termo-mesomediterráneas iberomarroquí-atlánticas (Ficario-Fraxinetum angustifoliae) ocupan en nuestro territorio los márgenes de los ríos sobre fluvisoles con un horizonte superior arenoso, están pseudogleizados en todo su perfil y rara vez son inundados. Si la textura del suelo se torna arcillosa la fresneda es sustituída por una chopera. El contacto con las saucedas se pone de manifiesto mediante la subasociación salicetosum pedicellatae y en los fluvisoles gravosos es sustituída por la subasociación tamaricetosum africanae. Como indicaron Ceballos y Martín Bolaños (1930), en el sector Aljíbico existe una agrupación mixta de fresnos con acebuches motivada por la presencia de capas freáticas en los bujeos y que llevamos a la subasociación Tamo-Oleetum fraxinetosum angustifoliae descrita por Benabid (1984) (lectotypus: tabla 2, inv. 3 , designado aquí) en el norte de Africa.

7f. Serie edafohigrófila aljíbico-tingitana del brezo ciliado (Erica ciliaris). Genisto anglicae-Ericeto ciliaris sigmetum

La vegetación turfófila permanente de 
brezales higrófilos (Genisto-Ericetum ciliaris) se instala en el dominio de las alisedas, saucedas y fresnedas, cuando la capa freática se encuentra muy próxima a la superficie del suelo y hay un gran aporte de materia orgánica. En el sector Aljíbico, esta serie se presenta como un mosaico de estos brezales y juncales (Juncetum rugoso-effusi, Junco emmanuelisEleocharitetum multicaulis). Rivas-Martínez et al. (1980) describieron el Erico ciliarisUlicetum lusitanici de las depresiones arenosas de Doñana, pero sus especies características (Centaurea uliginosa, Ulex minor var. lusitanicus) no llegan hasta nuestro territorio, por esta razón hemos llevado estos brezales higrófilos al Genisto-Ericetum descrito por Quézel et al. (1988) del sector Tingitano.

\section{7g. Serie edafohigrófila tingitano-onubo-} algarviense y bética del taraje africano (Tamarix africana). Polygono equisetiformisTamariceto africanae sigmetum

Polygono-Tamaricetum africanae es una asociación termomediterránea, bética y tingitano-onubo-algarviense, caracterizada por especies del género Tamarix, adelfas, y de otras plantas compañeras pertenecientes a la vegetación adyacente. Esta comunidad ocupa suelos arenosos, arcillosos o gravosos de cauces con largos períodos de sequía y que en épocas lluviosas son inundados soportando fuertes corrientes que erosionan y movilizan grandes cantidades de sedimento. En los principales ríos de nuestro territorio (subsectores Sidonense y Marbellí), los tarajales se presentan de forma apreciable alternando con las fresnedas y choperas. El contacto con las fresnedas viene dado por la subasociación aretosum italici, descrita por Rivas-Martínez et al. (1980) (lectotypus: tabla 61, inv. 5, designado aquí) en Doñana.

7h. Serie edafohigrófila ibérica del taraje (Tamarix canariensis). Agrostio stoloniferaeTamariceto canariensis sigmetum
Agrostio stoloniferae-Tamaricetum canariensis es una asociación constituída por bosquetes cerrados de tarajes que se instalan sobre suelos húmedos salobres e inundados hasta bien entrado el verano. Estos tarajales prefieren estaciones de vaguada, cubetas endorreicas o meandros abandonados, que drenan mal. Como etapa subserial aparecen asociados a estos tarajales los juncales de Holoschoeno-Juncetum acuti y de GalioJuncetum maritimi en función del gradiente de salinidad y del encharcamiento del suelo.

7i. Serie edafohigrófila ibérica de la adelfa (Nerium oleander). Rubo ulmifolii-Nerieto oleandri sigmetum

Rubo-Nerietum oleandri es una comunidad freatófita de adelfas y zarzas de amplia distribución ibérica que en nuestro territorio aparece distribuída por los subsectores Marbellí y Algecireño, siendo muy frecuente en las zonas bajas de los arroyos con fuerte desecación estival. Aparece generalmente en mosaico con juncales de Molinio-Holoschoenion.

\section{Las comunidades rupícolas}

El sector Aljíbico presenta un gran número de especies rupícolas endémicas ó de areales restringidos, lo que ha motivado la descripción de varias comunidades glerícolas y fisurícolas características del sector (Asensi, 1984; Galán de Mera, 1993; Deil, 1994; Galán de Mera et al., 1995a; Pérez Latorre et al. 1995 y Gil Giménez et al., 1996)

En los acantilados marítimos del subsector Algecireño encontramos la asociación Limonietum emarginati de areal AljíbicoTingitano. En éste mismo subsector pero en laderas margosas expuestas al mar se desarrolla la asociación Leontodonto tingitaniReichardietum picroidis, que se encuentra también en la Península Tingitana. En las calizas del distrito Gibraltareño (subsector Algecireño) se localizan tres comunidades 
distribuídas también por la península Tingitana, Biscutello sempervirentis-Iberidetum gibraltaricae, propia de acantilados calizos soleados, la com. de Campanula velutina y Chaenorrhinum villosum,que coloniza las oquedades sombrías de los roquedos y la com. de Silene tomentosa que se presenta de forma relíctica y muy empobrecida en los acantilados calizos. Incluímos estas tres últimas comunidades en Tinguarrenalia siculae Galán de Mera stat. nov y se le asigna el status de suborden ya que existen taxones en Andalucía y Norte de Africa que exceden el areal de Tinguarralia siculae, perteneciendo entonces a Asplenietalia petrarchae.

El esquema sintaxonómico para éste suborden en el sector Aljíbico sería el siguiente:

ASPLENIETEA TRICHOMANIS (Br.-Bl. in Meier \& Br.-B1. 1934) Oberdorfer 1977

Asplenietalia petrarchae Br.-B1. \& Meier 1934

Tinguarrenalia siculae (Daumas, Quézel \& Santa 1952) Galán de Mera stat. nov.

Campanulion velutinae Martínez Parras y Peinado Lorca 1990

Biscutello sempervirentis-Iberidetum gibraltaricae Deil 1994

com. Campanula velutina y Chaenorrhinum villosum com. Silene tomentosa

Del subsector Aljíbico es característica la comunidad relicta de Mucizonia hispida y Cheilanthes guanchica propia de areniscas con inclusiones calizas. En el subsector Marbellí se localiza la comunidad de Dianthus lusitanus caracterizada por caméfitos y hemicriptófitos que colonizan grietas en paredes de rocas silíceas (micaesquistos y gneises) soleadas; y finalmente la asociación Rupicapnetum africanae, de areal suribérico y norteafricano, espeluncícolo-nitrófila, basófila y termófila.

Entre las comunidades de amplio areal en el sector Aljíbico se encuentran: Sedetum micrantho-sediformis (subsectores Marbellí y Aljíbico), formada por pastizales rupícolas calcícolas con plantas crasas; Davallio
canariensis-Sedetum baetici, que es frecuente en todo el sector y está constituída por vegetación cormofítica, brio-pteridofítica de grietas anchas, sombrías y húmedas que en el subsector Algecireño aparece como subasociación psilotetosum nudi; por último, aparece también la asociación SelaginelloAnogrammetum leptophyllae, caracterizada por pequeños helechos y que coloniza la fisuras anchas y umbrosas de la base de rocas ácidas, apareciendo en el subsector Aljíbico y Algecireño como subasociación arisaretosum proboscidei.

\section{La vegetación psammófila}

Este tipo de vegetación edafoxerófila se desarrolla sobre los arenales litorales de los subsectores Marbellí y Algecireño, distribuyéndose catenalmente en función del estado de movilidad de las arenas y mayor o menor cercanía al mar (Díez et al., 1975; Gil et al., 1996).

9a. Serie psammófila tingitano-onuboalgarviense de la sabina Osyrio quadripartitae-Junipereto turbinatae sigmetum

En las dunas estabilizadas y edafizadas del subsector Marbellí se desarrolla la serie de los sabinares, cuya cabecera de serie (OsyrioJuniperetum turbinatae) se encuentra en mosaico con matorrales de Coremion albi.

Como vegetación climácica y en disposición catenal hacia el mar, encontramos en los valles interdunares un matorral incluíble en el Crucianelletum maritimae, mientras que en las crestas de las dunas estabilizadas aparece el Loto cretici-Ammophiletum australis y en las dunas embrionarias la asociación Cypero mucronati-Agropyretum juncei. Por último, se desarrolla la vegetación anual y pionera de arenas con materia orgánica del Salsolo kaliCakiletum maritimae.

Como etapas subseriales de las comunidades anteriores podemos reconocer a 
la comunidad terofítica de Ononido variegataeLinarietum pedunculatae y la comunidad anual nitrófila Sporobolo-Centaureetum sphaerocephalae.

\section{Las comunidades de marisma}

Están escasamente representadas en el sector Aljíbico, apareciendo fragmentariamente en las marismas de los ríos Palmones y Guadarranque (subsector Algecireño) las asociaciones Halimiono portulacoidis-Sarcocornietum alpini en la banda interior mas seca de las marismas e Inulo crithmoidis-Arthrocnemetum macrostachyi, que caracteriza a los niveles elevados arcillosos de los esteros litorales.

\section{Las comunidades dulceacuícolas}

La vegetación anfibia y acuática del sector Aljíbico es muy variada y comprende varias clases fitosociológicas. Como vegetación anfibia terofítica aparecen las asociaciones Laurentio-Juncetum tingitani sobre suelos arenosos poco cohesionados, ácidos y con aguas corrientes efímeras y superficiales, Loto subbiflori-Chaetopogonetum fasciculati de suelos arenosos o limo-arenosos de charcos o vaguadas con aguas superficiales durante el invierno y la primavera, Pulicario paludosaeAgrostietum pourretii de suelos areno-limosos con hidromorfía temporal, Cypero michelianiHeleochloetum alopecuroidis de los bordes de depresiones y embalses que permanecen encharcados hasta comienzos del verano y Glino lotoidis-Verbenetum supinae que contacta con la anterior eligiendo medios menos húmedos y arenosos.

Dentro de la vegetación geófita efímera aparece la asociación Junco pygmaei-Isoetetum velati dominada por Isoetes velatum y propia del borde areno-limoso de fosas profundas.

Como vegetación anfibia vivaz constituída por pequeños helófitos e isoétidos se presenta la asociación Junco emmanuelisEleocharitetum multicaulis responsable de la formación de un sustrato higroturboso, a veces también con especies de esfagnos (Sphagnum sp.).

La vegetación helofítica de aguas mesoeutróficas está representada por los cañaverales y espadañares de ríos y acequias del TyphoSchoenoplectetum tabernaemontan y por el Scirpetum maritimo-littoralis de aguas salobres.

De la vegetación acuática de hidrófitos constantemente enraizados destacamos las comunidades con Potamogeton nodosus en cubetas arcillosas profundas, Potamogeton pectinatus en aguas profundas y salobres, Myriophyllum alterniflorum en cubetas con aguas profundas oligótrofas, Callitriche stagnalis en pequeñas corrientes que se secan rápidamente a principios del verano, Callitriche regis-jubae en suelos ácidos por donde discurren aguas distróficas superficiales y Ranunculus tripartitus en medios higroturbosos.

La vegetación dulceacuícola cosmopolita de pequeños pleustófitos está representada por la comunidad de Lemna minor y la vegetación de carófitos bentonicos constituye la asociación Charetum vulgaris de aguas oligomesótrofas de profundidad variable.

\section{ÍNDICE SINTAXONÓMICO DE LAS ASOCIACIONES Y COMUNIDADES TRATADAS EN EL TEXTO}

QUERCETEA ILICIS Br.-B1. 1947

Myrto communis-Quercetum suberis Barbéro, Quézel \& Rivas-Martínez 1981 quercetosum suberis halimietosum halimifolii Pérez Latorre, Nieto Caldera y Cabezudo 1993

Teucrio baetici-Quercetum suberis Rivas-Martínez in Díez Garretas, Cuenca y Asensi 1988 quercetosum suberis quercetosum canariensis Asensi y Díez Garretas in Díez Garretas, Cuenca y Asensi 1988 
quercetosum rotundifoliae Nieto Caldera, Pérez Latorre y Cabezudo 1990

Tamo communis-Oleetum sylvestris Benabid 1984

\section{lectotipificada}

oleetosum sylvestris

quercetosum suberis Pérez Latorre, Nieto Caldera y Cabezudo 1993

fraxinetosum angustifoliae Benabid 1984 lectotipificada

Rusco hypophylli-Quercetum canariensis Rivas

Goday y Rivas-Martínez ex Rivas-Martínez 1974 quercetosum canariensis

rhododendretosum baetici Asensi y Díez Garretas in Díez Garretas, Cuenca y Asensi 1986

lauretosum nobilis Nezadal, Deil \& Welss 1994

quercetosum broteroi Pérez Latorre y Cabezudo subass. nova

Asparago aphylli-Calicotometum villosae Rivas-

Martínez 1975

calicotometosum villosae

juniperetosum turbinatae subass. nova

genistetosum linifoliae Pérez Latorre, Nieto

Caldera y Cabezudo 1993

Asparago albi-Rhamnetum oleoidis Rivas Goday 1959

rhamnetosum oleoidis

ericetosum scoparii Pérez Latorre, Nieto

Caldera y Cabezudo 1993

Clematido cirrhosae-Ceratonietum siliquae

Barbéro, Quézel y Rivas-Martínez 1981

phlomidetosum purpureae Martín Osorio, Díez

Garretas y Asensi 1991

Cytiso baetici-Arbutetum unedonis Nieto Caldera,

Pérez Latorre y Cabezudo 1990

arbutetosum unedonis

quercetosum cocciferae Nieto Caldera, Pérez

Latorre y Cabezudo 1990

bupleuretosum fruticosi Pérez Latorre, Nieto

Caldera y Cabezudo 1993

com. de Juniperus oxycedrus

Osyrio-Juniperetum turbinatae Rivas Martínez 1975

com. de Quercus lusitanica e Ilex aquifolium

QUERCO-FAGETEA Br.-Bl. \& Vlieger in Vlieger 1937

Cytiso triflori-Quercetum pyrenaicae Barbéro,

Quézel \& Rivas-Martínez 1981

Ranunculo ficariae-Fraxinetum angustifoliae Rivas-
Martínez y Costa in Rivas-Martínez, Costa, Castroviejo y Valdés 1980

fraxinetosum angustifoliae

salicetosum pedicellatae Díez Garretas, Cuenca y Asensi 1986

tamaricetosum africanae (Rivas-Martínez et al. 1980) Fernández González y Molina 1988

Crataego brevispinae-Populetum albae Galán de

Mera ass. nova

Frangulo baeticae-Rhododendretum baetici Rivas

Goday, Galiano y Rivas-Martínez ex Rivas-

Martínez, Fuente y Sánchez Mata 1986

rhododendretosum baetici

nerietosum oleandri Rivas Goday ex Asensi y

Díez Garretas in Díez Garretas, Cuenca y

Asensi 1986

Arisaro proboscidei-Alnetum glutinosae Martínez

Parras y Peinado Lorca 1987

alnetosum glutinosae

nerietosum oleandri Martínez Parras y Peinado Lorca 1987

Equiseto telmateiae-Salicetum pedicellatae Díez

Garretas, Cuenca y Asensi 1986

salicetosum pedicellatae

salicetosum albae Pérez Latorre y Cabezudo subass. nova

Rubo-Coriarietum myrtifoliae O. Bolós 1954

Lonicero hispanicae-Rubetum ulmifolii Rivas-

Martínez, Costa, Castroviejo y Valdés 1980

TRIFOLIO-GERANIETEA SANGUINEI Th.

Müller 1962

Calamintho baeticae-Galietum scabri Pérez Latorre,

Nieto Caldera y Cabezudo 1993

galietosum scabri

lamietosum flexuosi Pérez Latorre, Nieto Caldera y Cabezudo 1993

digitaletosum bocquetii Pérez Latorre, Nieto Caldera y Cabezudo 1993

NERIO-TAMARICETEA Br.-Bl. y O. Bolós 1957 Rubo ulmifolii-Nerietum oleandri O. Bolós 1956

Polygono equisetiformis-Tamaricetum africanae

Rivas-Martínez y Costa in Rivas-Martínez,

Costa, Castroviejo y Valdés 1980

tamaricetosum africanae

aretosum italici Rivas-Martínez, Costa,

Castroviejo y Valdés 1980 lectotipificado

Agrostio stoloniferae-Tamaricetum canariensis

Cirujano 1981 
CALLUNO-ULICETEA Br.-BI. \& R. Tx. 1943

Genisto tridentis-Stauracanthetum boivinii Rivas-

Martínez 1979

stauracanthetosum boivinii

quercetosum lusitanicae Pérez Latorre, Nieto

Caldera y Cabezudo 1993

ulicetosum baetici Pérez Latorre, Nieto

Caldera y Cabezudo 1993

Stauracantho boivinii-Drosophylletum lusitanici

Quézel, Barbéro, Benabid, Loisel \& Rivas-

Martínez 1988

saturejetosum salzmannii Galán de Mera y

Vicente Orellana 1996

Genisto anglicae-Ericetum ciliaris Quézel, Barbéro,

Benabid, Loisel \& Rivas-Martínez 1978

CISTO-LAVANDULETEA Br.-Bl. (1940) 1952

Calicotomo villosae-Genistetum lanuginosae

Martínez Parras, Peinado y de la Cruz 1987

corregido

genistetosum lanuginosae

genistetosum umbellatae Martínez Parras,

Peinado y de la Cruz 1987

genistetosum triacanthi Pérez Latorre, Nieto

Caldera y Cabezudo 1993

Cisto salvifolii-Ulicetum australis Pérez Latorre,

Nieto Caldera y Cabezudo 1993

ROSMARINETEA OFFICINALIS Br.-B1. $1931 \mathrm{em}$.

Rivas-Martínez, Díaz, Prieto, Loidi y Penas 1991

Asperulo hirsutae-Ulicetum scabri Díez Garretas,

Asensi y Fernández González 1995

ulicetosum scabri

anthyllidetosum cytisoidis Galán de Mera subass. nova

Teucrio lusitanici-Coridothymetum capitati Rivas

Goday y Rivas-Martínez 1968

MOLINIO-ARRHENATHERETEA R. Tx. 1937

Juncetum rugoso-effusi Rivas-Martínez y Costa in Rivas-Martínez, Costa, Castroviejo y Valdés 1980

Holoschoeno-Juncetum acuti Rivas-Martínez y Costa in Rivas-Martínez, Costa, Castroviejo y Valdés 1980

Galio palustri-Juncetum maritimi Rivas-Martínez y Costa in Rivas-Martínez, Costa, Castroviejo y Valdés 1980
LITTORELLETEA Br.-Bl. \& R. Tx. 1943

Junco emmanuelis-Eleocharitetum multicaulis

Rivas-Martínez y Costa in Rivas-Martínez, Costa, Castroviejo y Valdés 1980

PHRAGMITIO-MAGNOCARICETEA Klika in Klika \& Nowak 1941

Caricetum mauritanicae Díez Garretas, Cuenca y Asensi 1986

Typho-Schoenoplectetum tabernaemontani Br.-Bl. y O. Bolós 1957

Scirpetum maritimi-littoralis Br.-B1. 1931

JUNCETEA BUFONII (Br.-Bl. \& R. Tx. 1943) Foucault 1988

Laurentio-Juncetum tingitani Rivas Goday y Borja 1968

Loto subbiflori-Chaetopogonetum fasciculati RivasMartínez, Costa, Castroviejo y Valdés 1980

Pulicario paludosae-Agrostietum pourretii Rivas Goday 1955

Cypero micheliani-Heleochloetum alopecuroidis Rivas Goday y Valdés in Rivas Goday 1970

Glino lotoidis-Verbenetum supinae Rivas Goday 1964

ISOETETEA VELATAE (Br.-B1. \& R. Tx. 1943) Foucault 1988

Junco pygmaei-Isoetetum velati Rivas Goday 1955

HELIANTHEMETEA ANNUAE (Br.-B1. in Br.-B1. et al. 1952) Rivas Goday y Rivas-Martínez 1963 em. Rivas-Martínez 1978

Anthyllido hamosae-Malcolmietum lacerae Rivas Goday 1957 em. Rivas-Martínez 1978

Malcomio-Vulpietum alopecuris Díez Garretas, Hernández y Asensi 1975

Ononido variegatae-Linarietum pedunculatae Díez Garretas, Asensi y Esteve 1977

Velezio rigidae-Astericetum aquaticae Rivas Goday 1964

Trifolio-Plantaginetum bellardii Rivas Goday 1957 Sedetum micrantho-sediformis O. Bolós y $\mathrm{R}$. Masalles in O. Bolós 1981

RUDERALI-SECALIETEA CEREALIS Br.-B1. 1936 
Trifolio pallidi-Vulpietum geniculatae Galán de Mera 1995

ASPLENIETEA TRICHOMANIS (Br.-Bl. in Meier \& Br.-B1. 1934) Oberdorfer 1977

Biscutello sempervirentis-Iberidetum gibraltaricae Deil 1994

com. Campanula velutina y Chaenorrhinum villosum com. Silene tomentosa

com. Mucizonia hispida y Cheilanthes guanchica

Rupicapnetum africanae Pérez Latorre, Cabezudo y

Nieto 1995

Davallio canariensis-Sedetum baetici Deil 1994 sedetosum baetici

psilotetosum nudi Galán de Mera, Hagen y Vicente Orellana 1995

Selaginello-Anogrammetum leptophyllae R. Molinier 1937

anogrammetosum leptophyllae arisaretosum proboscidei Deil 1994

com. de Dianthus lusitanus

CRITHMO-LIMONIETEA Br.-B1. 1947

Limonietum emarginati Asensi 1984

Leontodonto tingitani-Reichardietum picroidis Deil 1994

AMMOPHILETEA Br.-B1. \& R. Tx. 1943

Loto cretici-Ammophiletum australis (Rivas Goday y Rivas-Martínez 1958) Rivas-Martínez 1964

Cypero mucronati-Agropyretum juncei (KühnholtzLordat 1923) Br.-B1. 1933

CAKILETEA INTEGRIFOliaE R. Tx. \& Preising in R. Tx. 1950 corr. Rivas-Martínez, Costa y Loidi 1992

Sporobolo-Centaureetum sphaerocephalae Díez Garretas, Hernández y Asensi 1975

Salsolo kali-Cakiletum maritimae Costa y Mansanet 1981 corr. Rivas Martínez, Costa y Loidi 1992 Crucianelletum maritimae Br.-B1. (1931) 1933

SALICORNIETEAFRUTICOSAE Br.-Bl. \& R. Tx. 1943 Halimiono portulacoidis-Sarcocornietum alpini Rivas-Martínez y Costa 1984

Inulo crithmoidis-Arthrocnemetum macrostachyi

(Fontes 1945) Géhu \& Géhu-Franck 1977

THERO-SALICORNIETEA Pignatti ex R. Tx. in R.

Tx. \& Oberdorfer 1958 corr. R. Tx. 1974
POTAMETEA PECTINATI R. Tx. \& Preising 1942 com. Potamogeton nodosus

com. Potamogeton pectinatus

com. Myriophyllum alterniflorum

com. Callitriche stagnalis

com. Callitriche regis-jubae

com. Ranunculus tripartitus

LEMNETEA MINORIS W. Koch \& R. Tx. in R. Tx. 1955

Com. Lemna minor

CHARETEA FRAGILIS (Fuk. 1961) Krausch 1964 Charetum vulgaris W. Krause 1969

\section{BIBLIOGRAFÍA}

ASENSI, A. -1984- Limonietum emarginati (Crithmo-Limonion) nueva asociación para los sectores Gaditano y Tingitano. Doc. Phytosociol., 8:45-50.

BARBERO, M., P. QUÉZEL y S. RIVASMARTÍNEZ -1981- Contribution à l'étude des groupements forestiers et préforestiers du Maroc. Phytocoenol., 9(3): 311-412.

BARBERO, M. y P. QUÉZEL - 1994- Place, role and historic value of laurifolius elements in Western Mediterranean preforest and forest vegetation. Annali di Botanica, 52:81-134.

BENABID, A. -1984- Etude phytoecologique des peuplements forestiers et preforestiers du Rif Centro-Occidental (Maroc). Trav. Inst. Sci. Chérifien, Sér. Bot. 34: 1-64.

BOLÒS, O., J. VIGO, R.M. MASALLES y J.M. NINOT -1990- Flora manual dels Països Catalans.. Barcelona.

BRAUN-BLANQUET, J. -1923- L'origine et la développement des flores dans le Massif central de France. Paris.

CARAZO MONTIJANO, M. y C. FERNÁNDEZ LOPEZ -1994- Flora de Marruecos y Andalucía. Catálogo bibliográfico de las plantas vasculares. Jaén.

CASTROVIEJO, S., M. LAINZ, G. LÓPEZ GONZÁLEZ, P. MONTSERRAT, F. MUÑOZ GARMENDIA, J. PAIVA y L. VILLAR -1986/ 1993- Flora iberica, I-IV. CSIC. Madrid.

CEBALLOS, L. y M. MARTÍN BOLAÑOS - $1930-$ Estudio sobre la vegetación forestal de la provincia de Cádiz. Instituto Forestal de Investigaciones y Experiencias. Madrid. 
CEBALlOS, L. y C. VICIOSO -1933-. Estudio sobre la vegetación y la flora forestal de la provincia de Málaga. IX Inspección del I.C.O.N.A. Madrid.

DAHLGREN, R. y P. LASSEN -1972- Studies in the flora of Northern Morocco, I. Some poor fen communities and notes on a number of northern and atlantic plant species. Bot. Notiser, 125: 439-463.

DEIL, U. -1994- Felsgesellschaften beiderseits der Strasse von Gibraltar. Hoppea, Denkschr. Regensb. Bot. Ges., 55: 757-814.

DIDON, J., M., DURAND-DELGA \& J. KORNPROBST -1973- Homologies gèologiques entre les deux rives du détroit de Gibraltar. B.S.G.F., 15(7):76-104.

DÍEZGARRETAS, B., A.M. HERNÁNDEZy A. ASENSI -1975-Estructuras de algunas comunidades vegetales de dunas en el litoral de Marbella (Málaga). Acta Bot. Malacitana, 1:69-80.

DÍEZ GARRETAS, B., J. CUENCA y A. ASENSI 1986- Datos sobre la vegetación del subsector Aljíbico (provincia Gaditano-OnuboAlgarviense). Lazaroa, 9: 315-332.

DUPONT, P. -1962-Laflora atlantique europeenne. Documents pour les Cartes des Productions Végétales, I. Toulouse.

GALÁN DE MERA, A. -1993- Flora y vegetación de los términos municipales de Alcalá de los Gazules y Medina Sidonia (Cádiz, España). Tesis Doctoral. Universidad Complutense. Madrid.

GALÁN DE MERA, A. -1993- Silene tomentosa Otth (Caryophyllaceae) from Southern Spain, is not an extinct species. Rivasgodaya, 7: 119-126.

GALÁN DE MERA, A., M.A. HAGEN y J.A. VICENTE ORELLANA -1995a- Variabilidad de las comunidades rupícolas brio-pteridofíticas en el SO de la Península Ibérica y NO de Africa. Orsis, 11. (en prensa).

GALÁN DE MERA, A., J.A. VICENTE ORELLANA, J.L. GONZALEZ y J.C. FERNÁNDEZ LUNA - 1995b- New populations of Psilotum nudum in SW Europe (Psilotaceae: Pteridophyta). Fern Gaz. (en prensa).

GALÁN DE MERA, A. y J.A. VICENTE ORELLANA -1996- Phytosociological study of the plant communities with Stauracanthus boivinii of the SW of the Iberian Peninsula and NW of Africa, using multivariate analysis. Bot. Helv., 106:45-56.

GAUSSEN, H., J. DEBRACH \& F. JOLY -1958-
Précipitations Annuelles. Atlas du Maroc. Comité de Gèographie du Maroc. Rabat.

GÉHU, J.M. -1991-Végétations et paysages littoraux de type cantabro-atlantique. Ber. $d$. Reinh. Tüxen-Ges., 3: 59-128.

GIL, J.M., J. ARROYO y J.A. DEVESA -1985Contribución al conocimiento florístico de las sierras de Algeciras (Cádiz, España). Acta Bot. Malacitana, 10: 97-122.

GIL JIMÉNEZ, Y., P. NAVAS FERNÁNDEZ, D. NAVAS FERNÁNDEZ, A.V. PÉREZ LATORRE y J.M. NIETO CALDERA -1996Tipos de hábitats naturales contemplados en la directiva 92/43 C.E.E. presentes en el Campo de Gibraltar y espacios naturales de interés para su conservación. Almoraima, 15: 255-266.

GREUTER, W., H.M. BURDET y G. LONG -19841989-Med-Checklist. Conservatoire et Jardin Botaniques. Genève.

GUTIÉRREZ MAS, J.M., A. MARTÍN ALGARRA, S. DOMÍNGUEZ BELLA y J.P. MORAL CARDONA -1991- Introducción a la geología de la provincia de Cádiz. Universidad de Cádiz.

JAHANDIEZ, E. \& R. MAIRE -1931-1934Catalogue des plantes de Maroc. Alger.

LINARES, L., A. HARPER y J. CORTES -1996The flowers of Gibraltar. Flora Calpensis.

MAIRE, R. -1953-1987-Flore de l'Afrique du Nord, 1-16. P. Lechevalier. Paris.

MARTÍNEZ PARRAS, J.M. y M. PEINADO LORCA -1987- Datos sobre la vegetación riparia del sector Gaditano. Secret. Public. ser. Informes, 22: 199 206. Universidad de la Laguna.

MEUSEL, H., E. JAEGER \& E. WEINERT -1965Vergleichende Chorologie der Zentraleuropaischenflora. Jena.

NEZADAL, W., U. DEIL \& W. WELSS -1994Karte der aktuellen Vegetation des Campo de Gibraltar (Provinz Cádiz, Spanien). Hoppea, Denkschr. Regensb. Bot. Ges., 55: 717-756.

NIETO CALDERA, J.M., A.V. PÉREZ LATORRE y B. CABEZUDO -1990- Datos sobre la vegetación silicícola de Andalucía, I. Acta Bot. Malacitana, 15: 179192.

NIETO CALDERA, J.M., A. V. PÉREZ LATORRE y B. CABEZUDO - 1991- Biogeografía y series de vegetación de la provincia de Málaga (España) Acta Bot. Malacitana, 16(2): 417-436.

NIETO CALDERA J. M, B CABEZUDO Y A. V. PÉREZ LATORRE -1994- "Caracterización bioclimática de los alcornocales andaluces (series de vegetación)». Anais do Instituto 
Superior de Agronomía, 44: 269-283.

OBERDORFER, E. -1990- Pflanzensoziologische Excursionsflora. Stuttgart.

OJEDA, F., T. MARAÑÓN y J. ARROYO -1996Patterns of ecological, chorological and taxonomic diversity at both sides of the Strait of Gibraltar. Journal of Vegetation Science, 7: 6372.

PEINADO LORCA, M. y S. RIVAS-MARTÍNEZ 1987- La vegetación de España. Univ. Alcalá de Henares. Madrid.

PÉREZ LATORRE, A.V. -1993- Estudio fitocenológico de los alcornocales andaluces. Tesis Doctoral. Universidad de Málaga. Málaga. PÉREZ LATORRE, A.V., J.M. NIETO CALDERA y B. CABEZUDO -1993-Contribución al conocimiento de la vegetación de Andalucía. II. Los alcornocales. Acta Bot. Malacitana, 18: 223-258.

PÉREZ LATORRE, A.V., J.M. NIETO CALDERA y B. CABEZUDO -1994- Datos sobre la vegetación de Andalucía III. Series de vegetación caracterizadas por Quercus suber $\mathrm{L}$. Acta Bot. Malacitana, 19: 169-185.

PÉREZ LATORRE, A. V., B CABEZUDO y J.M NIETO -1995- Nota fitosociológica sobre Rupicapnos africana subsp, decipiens en el sur de España. Acta Bot. Malacitana, 20: 310-311

PICHI SERMOLLI, R.E.G., L. ESPAÑA y A.E. SALVO -1988- El valor biogeográfico de la pteridoflora ibérica. Lazaroa, 10: 187-205.

PICHI SERMOLI, R.E.G. -1991- Cosiderazioni sull'affinitá ed origine della flora pteridologica della Regione Mediterranea. Acta Bot. Malacitana, 16(1):235-280.

QUÉZEL, P. -1957-Peuplement végétal des hautes montagnes de l'Afrique du Nord. Paris.

QUÉZEL, P. -1978- Analysis of the flora of Mediterranean and Saharan Africa. Ann. Missouri Bot. Gard., 65: 479-534.

QUÉZEL, P., M. BARBERO, A. BENABID, R. LOISEL y S. RIVAS-MARTÍNEZ -1988Contribution à l'étude des groupements préforestiers et des matorrals rifains. Ecol. Medit., 14(1/2): 77-122.

REFASS, M. -1993-Tanger. Espace, économie et société. Université Mohammed V, Rabat. Université Abdelmalek Es-Saadi, Tanger.

RIVAS GODAY, S., E. FERNÁNDEZ GALIANO Y S. RIVAS MARTÍNEZ -1965-. Vegetación natural in: Estudio agrobiológico de la provincia de Cádiz. I. N. I. A. Sevilla.
RIVAS GODAY, S. -1967-Algunos aspectos de la vegetación gaditana. Cádiz.

RIVAS GODAY, S. -1968- Algunas novedades fitosociológicas de la España meridional. Collect. Bot., Barcelona, 7: 997-1031.

RIVAS-MARTÍNEZ, S., M. COSTA, S. CASTROVIEJO y E. VALDES -1980Vegetación de Doñana (Huelva, España). Lazaroa, 2: 5-189.

RIVAS-MARTÍNEZ, S. -1987-Memoria y mapa de las series de vegetación de España (1: 400.000). ICONA. Madrid.

RIVAS-MARTÍNEZ, S. -1988- Bioclimatología, biogeografía y series de vegetación de Andalucía Occidental. Lagascalia, 15: 91-119.

RIVAS-MARTÍNEZ, S., M. LOUSA, T.E. DÍAZ, F. FERNÁNDEZ GGNZÁLEZ y J.C. COSTA 1990- La vegetación del sur de Portugal. Itinera Geobot., 3: 5-126.

RIVAS-MARTÍNEZ, S., A. ASENSI, J. MOLERO MESA y F. VALLE -1991- Endemismos vasculares de Andalucía. Rivasgodaya, 6: 5-76.

TAKHTAJAN, A.L. -1986-Floristic regions of the world. University of California Press. Berkeley. Los Angeles. London.

TUTIN, T., V.H. HEYWOOD, N.A. BURGES, D.M. MOORE, D.H. VALENTINE, S.M. WALTERS y D.A. WEBB -1964-1980- Flora Europaea. Cambridge University Press.

VALDÉS, B., S. TALAVERA y E.F. GALIANO 1987- Flora vascular de Andalucía Occidental. Barcelona.

VALDÉS, B. 1996. El elemento endémico del Campo de Gibraltar. Almoraima, 15: 13-34.

WILLKOMM, H.M. y J.M. LANGE -1861-1880Prodromus florae hispanicae, I-III. Stuttgart.

WOJTERSKI, T.W. -1985-Guide de l'excursion internationale de phytosociologie. Algerie du Nord. El Harrach.

Aceptado para su publicación en Junio de 1996.

Dirección de los autores. B. CABEZUDO y A.V. PÉREZ LATORRE: Dpto. Biología Vegetal, Facultad de Ciencias, Universidad de Málaga, E29071 Málaga (España). U. DEIL: Lehrstuhl Biogeographie, Universität Bayreuth, D-95440 Bayreuth (Alemania). A. GALAN DE MERA: Lab. Botánica, Universidad San Pablo-CEU, Ap. 67, E28660 Boadilla del Monte. Madrid (España). 